livraisons

d'Histoire

de l'Architecture

\section{Livraisons de l'histoire de l'architecture}

14 | 2007

Piscines

\title{
Les baignades en rivière d'île-de-France, des premiers aménagements à la piscine parisienne Joséphine-Baker
}

Swimming in the river of the Ile-de-France: from the first facilities to the Josephine Baker swimming pool

Flussbadeanstalten in der Île-de-France, von den ersten Einrichtungen zu dem Schwimmbad Josephine Baker

Isabelle Duhau

\section{(2) OpenEdition}

Journals

Édition électronique

URL : http://journals.openedition.org/lha/422

DOI : $10.4000 /$ /ha. 422

ISSN : $1960-5994$

Éditeur

Association Livraisons d'histoire de l'architecture - LHA

Édition imprimée

Date de publication : 10 décembre 2007

Pagination : 9-38

ISSN : 1627-4970

Référence électronique

Isabelle Duhau, "Les baignades en rivière d'Île-de-France, des premiers aménagements à la piscine parisienne Joséphine-Baker », Livraisons de l'histoire de l'architecture [En ligne], 14 | 2007, mis en ligne le 10 décembre 2009, consulté le 30 avril 2019. URL : http://journals.openedition.org//ha/422 ; DOI :

$10.4000 /$ /ha. 422

Ce document a été généré automatiquement le 30 avril 2019.

Tous droits réservés à l'Association LHA 


\title{
Les baignades en rivière d'île-de- France, des premiers aménagements à la piscine parisienne Joséphine- Baker $^{1}$
}

\author{
Swimming in the river of the Ile-de-France: from the first facilities to the \\ Josephine Baker swimming pool \\ Flussbadeanstalten in der Île-de-France, von den ersten Einrichtungen zu dem \\ Schwimmbad Josephine Baker
}

Isabelle Duhau

«En général, tout établissement de bains sur les eaux ne peut manquer d'avoir une grande supériorité sur ceux construits dans l'intérieur d'une grande ville. L'illusion de la belle nature peut-elle jamais naitre au milieu de ces petits jardins factices, circonscrits dans un étroit terrain? Vous n'avez pas quitté la fatigue des rues, le bruit des cabriolets, des fiacres, la scène n'a pas changé : au lieu que dans les bains sur la Seine, c'est un nouvel air que vous respirez, un nouveau monde sur la plage duquel vous abordez, en laissant sur la rive, comme un souvenir importun, les idées sombres et mélancoliques qui ne cessent de vous obséder dans les fracas de la ville ${ }^{2}$.» Si l'homme se baigne depuis la nuit des temps en milieu naturel, la construction d'équipements spécifiques sur les rives de nos cours d'eau ou de nos lacs ne semble pas remonter à plus de trois siècles. Le bain public est une pratique courante dans les mondes antique ou arabe ; ceux-ci ont construit des édifices publics à cet usage au cœur de leurs villes. Notre imaginaire évoque sans difficulté les thermes romains ou les hammams orientaux. Cependant, en Occident, la tradition du bain avait disparu (seul le bain thérapeutique restait quelquefois prescrit), la religion chrétienne rejetant la nudité et édictant des règles de pudeur strictes. Le thème mythologique de Venus au bain sert de prétexte aux artistes, dans la tradition classique, pour représenter le corps féminin nu dans un paysage champêtre. À Paris, au XIII ${ }^{\mathrm{e}}$ siècle, on trouve le long de la Seine de rares installations stables d'étuves dont on ne connaît pas les caractéristiques architecturales 
et qui disparaissent à la Renaissance, l'usage des pommades et les onguents venus d'Italie devenant la règle de la toilette. Pour ceux qui aiment l'eau, il ne reste que le bain en rivière. Le mois d'août des Très riches heures du duc de Berry (musée Condé, Chantilly) montre quelques baigneurs s'ébattant dans la Juine, tout près d'Etampes. On sait que le roi Henri IV était friand de ces bains froids qu'il pratiquait à Saint-Germain tandis que Louis XIV se baignait également à Valvins ${ }^{3}$. À partir du XVII e siècle, les Parisiens sont plus nombreux à se baigner dans la Seine, comme à la porte Saint-Bernard ${ }^{4}$, mêlant un souci d'hygiène au plaisir rafraîchissant de l'eau, aux beaux jours. Leur nudité troublant l'ordre public, la nécessité d'installations spécifiques s'impose, la baignade sauvage demeurant la règle partout ailleurs dans les lieux isolés. Les établissements de bains se multiplient au XVIII ${ }^{e}$ siècle, le naturel et l'exercice physique étant mis en avant par les nouvelles théories des Lumières et les médecins se montrant de plus en plus favorables aux bains. Ces institutions proposent baignoires et bassins collectifs mais il faut attendre la fin du siècle pour qu'apparaissent les premières écoles de natation. Dès lors, les établissements au bord de nos rivières se multiplient afin de répondre à la demande d'une pratique sportive naissante, tandis que le bain d'hygiène se replie peu à peu dans la sphère privée ${ }^{5}$.

1 Les évolutions techniques et architecturales des équipements répondent à leurs évolutions fonctionnelles. D'abord structures rudimentaires, les baignades se perfectionnent afin de garantir davantage de sécurité aux nageurs (cloisonnement pour réguler le courant, fond artificiel en pente douce), de leur proposer plus de confort (vestiaires individuels, location de serviettes et de costumes), des services complémentaires (espaces de repos, restaurants, salons privés ou dancings) ou bien encore des aménagements ludiques (plages, toboggans ou plongeoirs). Les établissements flottants, installés pour la belle saison et remisés l'hiver, côtoient ceux devenant définitifs au fur et à mesure que leurs équipements se développent, que les rivages sont aménagés ou que les constructions en béton se substituent aux premiers bains en bois. Paris, pionnière en matière de baignades en rivière, se voit à partir de la seconde moitié du XIX siècle peu à peu distancée par les nombreuses installations des rivages franciliens suscitées par la mode de la partie de campagne dominicale. Dans l'entre-deux-guerres, les baignades, de plus en plus sophistiquées, deviennent de véritables établissements nautiques polyvalents baptisés "plages ", rivalisant avec les établissements balnéaires attirant la haute société dans des villégiatures luxueuses. Tandis que les premiers bains en eau vive étaient nés d'une réhabilitation de l'eau dans l'hygiène et la santé corporelles, la nudité des corps dans l'espace urbain ainsi que la sécurité des baigneurs constituent des problèmes récurrents pour les pouvoirs publics, contraints à réglementer et surveiller strictement les baignades. Après guerre et jusqu'aux années 1960, grâce au développement des loisirs aquatiques, les baignades en rivière connaissent encore quelques saisons d'engouement, avant que la multiplication des piscines couvertes artificielles répondant aux besoins croissant de l'enseignement de la natation aux scolaires et de la pratique sportive, et surtout la pollution des rivières, n'oblige les établissements à fermer définitivement leurs portes. 


\section{Installations démontables et structures pérennes}

\section{Limites lexicales}

2 Aucun terme spécifique ne qualifie les dispositions qui permettent les bains en eau vive, dits froids. On y pratique la natation dans ce que les textes mentionnent successivement ou alternativement comme des bains, des écoles de natation, des baignades, des plages ou des piscines. L'absence de définition pour ce lieu (qu'il borde une rivière ou qu'il soit totalement artificiel) dans le Grand dictionnaire universel du XIXe siècle de Pierre Larousse (1866) est symptomatique et atteste le caractère récent de l'encadrement de la baignade. Selon la dernière édition du Grand Robert de la langue française (2001), la piscine « est un grand bassin où l'on se baigne en commun " (l'expression "piscine de natation " apparaissant en 1865) ; le bain est défini entre autres comme «l'action d'entrer dans l'eau pour le plaisir et éventuellement dans l'intention d'y séjourner, de nager » tandis que la baignade «est à la fois l'action de se baigner en mer, en lac ou en rivière et l'endroit d'un cour d'eau ou d'un lac où l'on peut se baigner ». Cette dernière définition sous-entend que la baignade se pratique en plein air mais dans des lieux qui ne sont cependant pas nécessairement aménagés pour cet usage. Les techniciens décrivent ceuxci plus précisément. Selon eux ${ }^{6}$, les baignades sont des bassins naturels ouverts, des plages aménagées en bordures des rivages de la mer ou englobant des portions de cours d'eau, ou bien encore utilisant des lacs et des étangs naturels. Les bassins constituent des établissements de natation que l'eau traverse en circuit ouvert, partiellement naturel et partiellement artificiel. Enfin, les piscines comportent un bassin en plein air ou couvert et sont un aménagement totalement artificiel, fonctionnant en circuit fermé. Ainsi, aujourd'hui encore en Île-de-France, on se baigne en rivière dans quelques lieux plus ou moins façonnés par l'homme, de la crique naturelle dégagée de ses herbages aux installations techniques plus sophistiquées.

\section{Des bateaux - pontons...}

3 L'Encyclopédie explique que les bains sont un terme d'architecture correspondant à de grands et somptueux bâtiments. Elle distingue les bains artificiels de ceux dits naturels qui sont, ou chauds, comme ceux des eaux minérales, ou froids, comme l'eau des rivières. L'ouvrage ajoute qu'à cette date :

4 Les bains publics sur la rivière, ne sont autre chose que de grands bateaux, appelés toue ${ }^{7}$, faits de sapin, et couverts d'une grosse toile, autour desquels il y a de petites échelles attachées par des cordes, pour descendre dans un endroit de la rivière où l'on trouve des pieux enfoncés d'espace en espace, qui soutiennent ceux qui prennent le bain'

5 Ainsi, dans le bateau amarré à la rive, le public dépose ses vêtements, abrité sous la toile tendue, puis descend dans l'eau, toujours protégé des regards par la toile prolongée en auvent au-dessus de l'eau et maintenue par les pieux délimitant le bassin. Cependant, les gens de qualité ne fréquentent pas ces établissements. Pour les gens riches, les bateliers ont installé, au-dessus et au-dessous de Paris, des petites cabanes nommées gores qui se composent de quatre pieux ombragés par une toile; un autre pieu, planté au milieu, permet de se soutenir sur l'eau. Paul Négrier mentionne le Journal du citoyen selon lequel «les dames sont conduites et descendues dans ces gores, sûrement, commodément et 
secrètement »; Négrier précise que ce sont les femmes des mariniers qui guident ces baigneuses.

6 Vers 1780, le sieur Turquin ouvre un nouvel établissement sur le petit bras du fleuve parisien, près du pont de la Tournelle : il place dans son bateau des baignoires percées, maintenues sur un plancher à une certaine profondeur et traversées par le courant de la rivière. Trois personnes qui disposent de cabines pour se déshabiller peuvent prendre place dans ces baignoires. La maison est bientôt baptisée «bains chinois »". Devant le succès, Turquin ouvre un autre établissement en 1785 : il supprime les baignoires mais aménage un bassin collectif. L'entrepreneur crée encore un troisième bain au pont Royal. En 1808, il s'associe à son gendre Deligny. Turquin se fait l'ardent défenseur des bains froids et du concept d'école de natation ${ }^{10}$. Le terme est nouveau mais seule la terminologie change, il s'agit pour l'entrepreneur d'attirer une clientèle privilégiée qui dédaignait jusque là les bains collectifs. En effet, les aménagements évoluent finalement peu par rapport aux bains collectifs existants. L'ordonnance de police du 3 juin 1783 exige simplement le cloisonnement total du bain dans ses parties supérieures (afin d'éviter que les passants ne voient les baigneurs) et dans ses parties latérales (la sécurité des usagers imposant de se prémunir du courant). Toutefois devant le succès de ces équipements, les dimensions de ceux-ci augmentent et leur mise en place saisonnière tend à se muer en installation durable. Longtemps, les bateaux avaient été soumis aux aléas du dégel et étaient parfois endommagés voire coulés par des blocs de glace charriés par la rivière. Ils étaient remisés à l'abri durant la saison d'hiver, désencombrant les berges et le trafic fluvial.

7 Au début du XIX siècle, les bains établis sur la Seine, à Paris, se composent désormais de quatre ou six pontons portant plateforme, cabines, portiques, restaurant, qui forment ensemble une enceinte rectangulaire, au milieu de laquelle se prennent les bains. Les espaces annexes se développent, comme aux premiers bains Deligny où "la rotonde " appelée encore «l'amphithéâtre $»^{11}$ permet aux nageurs de se réunir et de discuter. À partir de 1830, le fond du bassin peut être formé de planches de bois mobiles susceptible de modifier la profondeur et de créer une pente artificielle. En 1840, les frères Burgh, nouveaux propriétaires, reconstruisent l'école de natation Deligny (ill. 1) :

Elle est formée par l'assemblage de plusieurs bateaux. Un bateau d'entrée contient le bureau de recette, la lingerie, le logement du gérant, et des cabinets dans sa partie supérieure. Un bateau-rotonde, placé à la tête de l'école, contient le café, sa cuisine et son divan; en aval, au bas de l'école, est un autre bateau-rotonde. Dix bateaux, rangés sur deux lignes, dans l'ordre d'un parallélogramme, achèvent l'enceinte de l'école. Ajoutons le bateau séchoir, qui sert de logement au gardien, et la buanderie flottante. Cet ensemble est décoré avec beaucoup de goût, de luxe, et atteste la légèreté, la fantaisie élégantes, les teintes vives, les nuances variées et les découpures de l'architecture orientale. Le divan est à lui seul un kiosque délicieux, et dans lequel la lumière du jour ne pénètre qu'à travers des verres de couleur qui en adoucissent l'éclat.

9 Trois cent quarante cabinets règnent le long des galeries du rez-de-chaussée et du premier étage: chacun est meublé de glaces, de patères, de tapis, de chaises en frêne couvertes en canne. Il y a, en outre, six salons particuliers, loués à l'année, sept salles communes, avec des cases pour les effets des baigneurs, six salles pour les pensions, et six autres salles à l'étage supérieur. Les galeries sont garnies d'un tapis de laine. Indépendamment de tous ces lieux ouverts au public, on a réservé trois cabinets avec onze cents cases numérotées pour le dépôt des objets précieux, une salle pour les leçons à 
sec, et une chambre de secours, munie des appareils nécessaires pour rappeler les noyers à la vie; et je ne parle pas des pièces de service, du salon de coiffeur et du salon de pédicure.

10 Le fond d'un des deux bassins a été dragué pour faire disparaittre toutes les aspérités du sol : l'autre bassin est muni d'un fond en bois, long de trente mètres, dont la profondeur va de soixante centimètres à deux mètres. Devant le bateau-divan se trouve un escalier en spirale à deux paliers, dont le second s'élève à six mètres au-dessus de l'eau. Cette montée, d'une construction élancée est entourée de filets : un mât et une flamme lui donnent un aspect pittoresque. Les plongeurs donnent à cet appareil qui leur est destiné le sobriquet de perchoir. Ils ont aussi, sur le pont qui joint les deux galeries, des gradins d'où ils peuvent prendre leur élan.

11 Les bains Deligny sont le prototype du genre ${ }^{12}$.

\section{1 : Les bains Deligny}

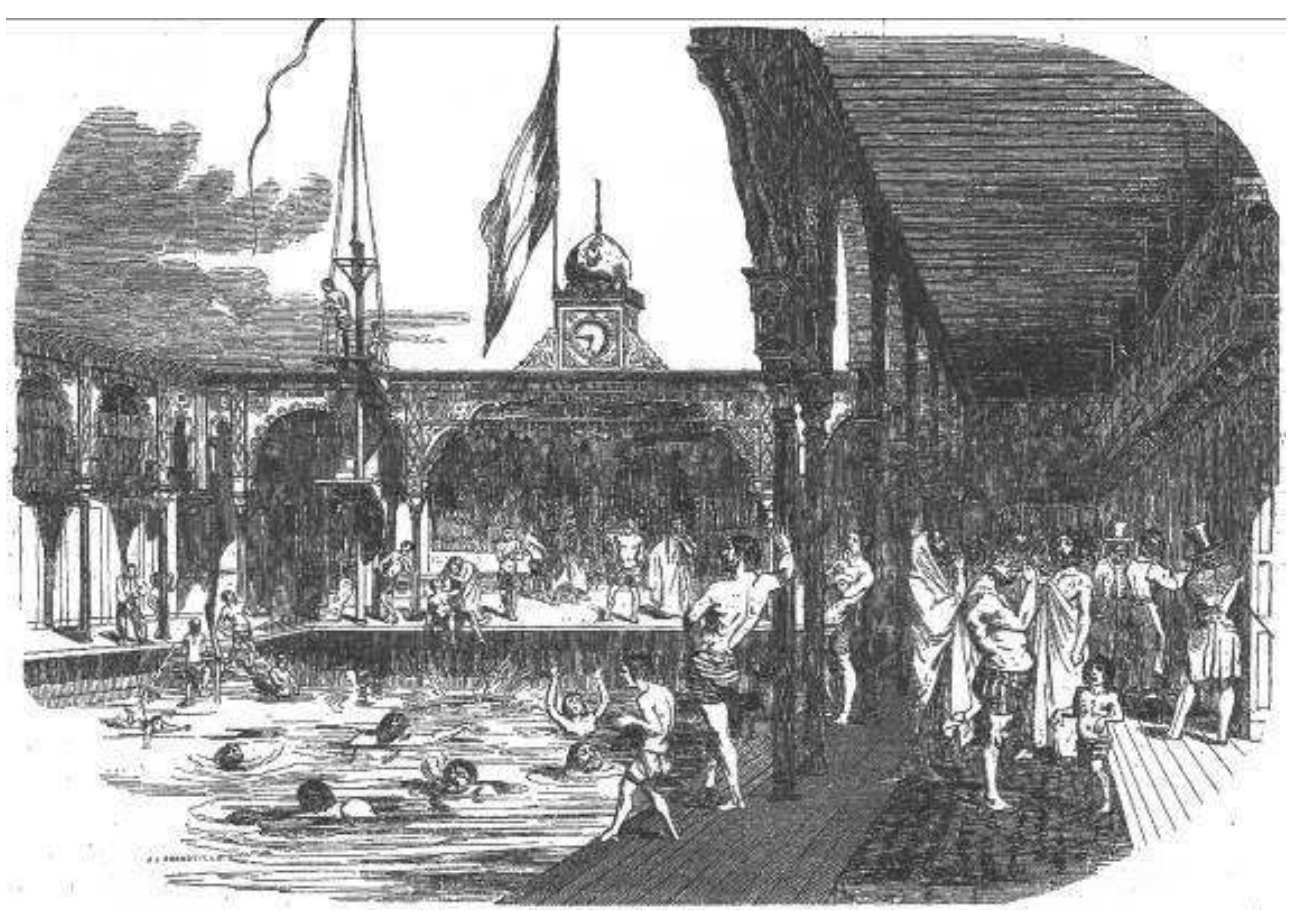

EDMOND TEXIER, OP. CIT., P. 5.

(C) Inventaire général, repro. Stéphane Asseline

Effectivement, durant des décennies, nombre d'établissements s'inspirent du modèle créé par l'architecte Galant (élève de Visconti) et les décorateurs Philastre et Cambon. Leur mise en scène orientalisante, style associé aux bains dès le XVIII ${ }^{e}$ siècle à Paris ${ }^{13}$, marque les esprits. Un confort matériel inédit est offert aux clients. Les bassins sont entourés de filets pour éviter aux nageurs de rejoindre la pleine eau et assurer ainsi leur sécurité. La pente progressive du premier bassin satisfait les nageurs débutants tandis que les plus expérimentés peuvent plonger dans le second bain, où le fond naturel de la rivière atteint ici 4,50 m. de profondeur. Enfin, les baigneurs profitent du ciel parisien car l'école est construite près de la rive, à l'abri d'un rideau d'arbres élevés et de grands peupliers et ne nécessite pas d'être recouverte d'une toile ${ }^{14}$. Près de cent ans plus tard, la réglementation atteste du peu d'évolution techniques des bassins parisiens qui sont toujours construits selon le même modèle (ill. 2 et 3) : 
13 1. Les bateaux devront toujours être en bon état et bien gibassés ${ }^{15}$ de manière qu'il n'y ait jamais d'eau en cale. Ils seront fréquemment nettoyés à fond pour éviter dépôts de vases ou d'ordures. Ils seront solidement amarrés.

14 2. Le fond en bois sera composé d'un plancher en charpente solidement boulonné, il ne sera pas cloué, mais maintenu au moyen de vis à tête ronde et noyée dans le bois.

15 3. Le périmètre interne du bain sera garni dans toute sa hauteur, ou de herses en charpente à claire-voie, dont les pièces ne seront pas écartées l'une de l'autre de plus de 0,15 ou de filets métalliques suffisamment forts dont les mailles auront au plus 0,15 de $\operatorname{coté}^{16}$.

III. 2 : Les bains parisiens

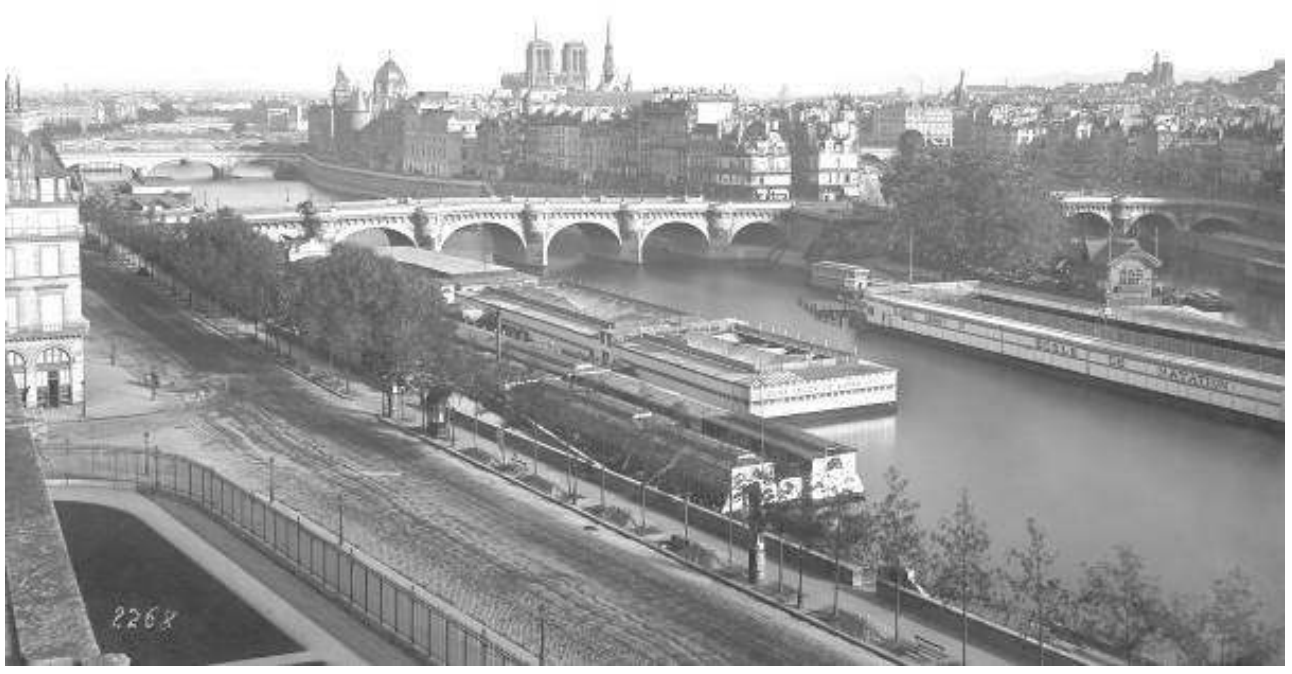

Photographiés à la fin du XIXe siècle par Armand Guérinet

(c) Ministère de la Culture, médiathèque du patrimoine, Archives photographiques 


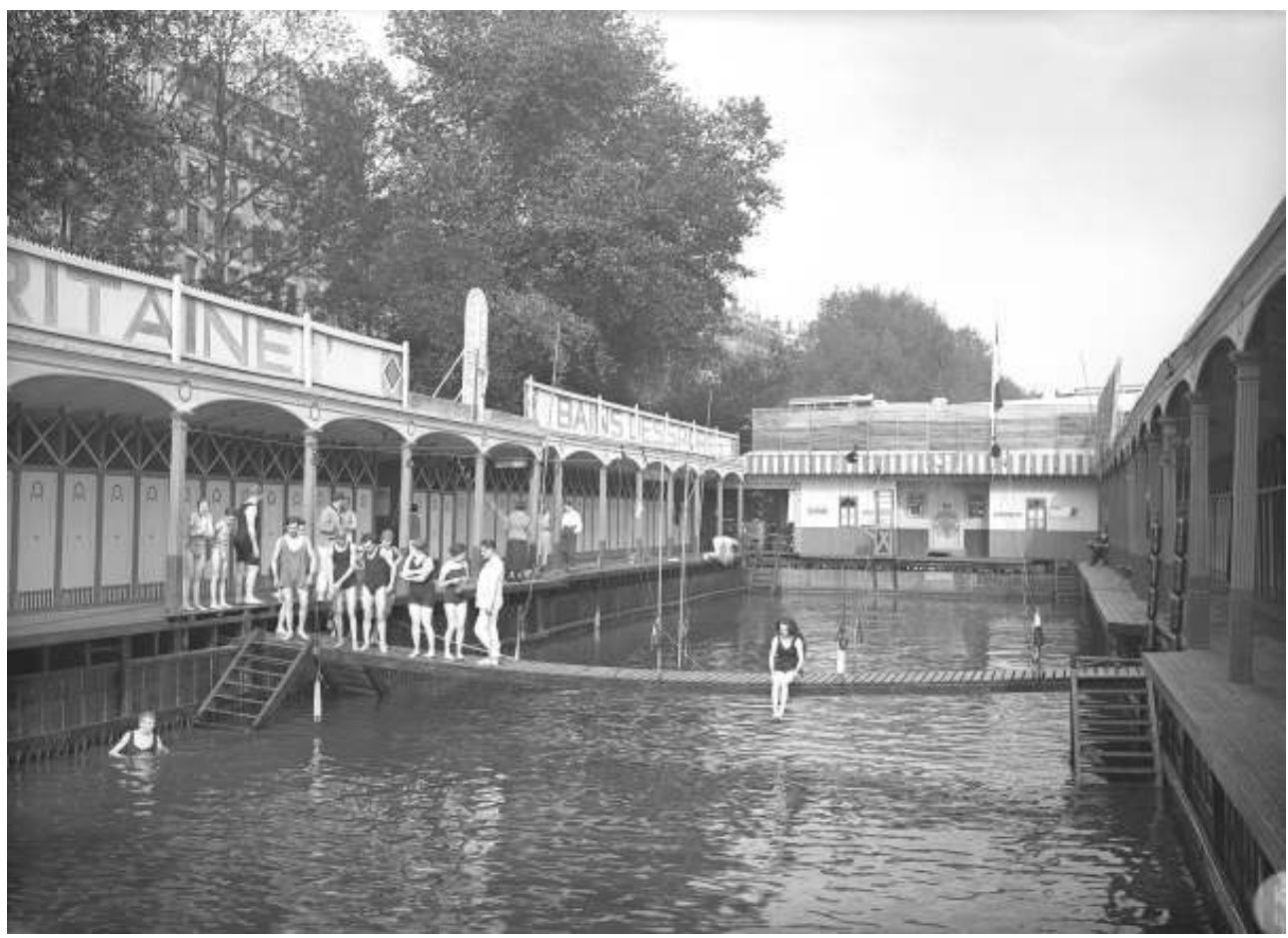

Photographiés par les frères Séeberger

(c) Ministère de la culture, médiathèque du patrimoine. Archives photographiques

Cependant, à partir de la fin du XIX siècle, les rivages des alentours de la capitale voient se développer une nouvelle typologie de baignades: tandis que le bassin demeure délimité par des pontons flottants, les aménagements annexes sont installés directement sur la berge. Ils complètent les guinguettes et autres ateliers de constructeurs de bateaux dont les propriétaires gèrent également des établissements de bains, la polyvalence de leurs activités étant gage de succès. Ces équipements complémentaires présentent les mêmes caractéristiques de précarité. Conçus en partie en bois, agrandis et reconstruits autant que de besoin, l'éclectisme et la fantaisie de leurs décors répondent aux aspirations de dépaysement de la clientèle recherchant l'évasion à leur porte. Les berges sont le plus souvent aménagées en conséquence mais les investisseurs exploitent, dès qu'elles existent, les richesses naturelles des rivages, tel le premier établissement de la plage de l'Isle-Adam, la plage de sable de Gournay-sur-Marne ou le banc de sable de Joinville-le-Pont ${ }^{17}$. Les évolutions formelles de ces bassins restent liées aux évolutions techniques. Longtemps bâtis tout en bois, les plus sophistiqués permettent un réglage de la hauteur du fond, suspendu à des chaînes.

Après la seconde guerre mondiale, les constructeurs initient une production industrielle, substituant le métal au bois et assemblant des modules préfabriqués les uns aux autres, permettant ainsi la fabrication de bassins de dimensions et de formes variées et facilitant leur démontage hivernal. Le bassin-école flottant que la société des constructions métalliques SES installe à Meaux, en 1951, donne toute satisfaction à la fois à la municipalité, au service technique de l'équipement du secrétariat d'État à l'enseignement technique, à la jeunesse et aux sports et à la fédération française de natation ${ }^{18}$. Bientôt la première loi-programme d'équipements sportifs (1962-1965) encourage la construction de bassins de natation et de piscines, les villes françaises demeurant largement sous 
équipées. La loi préconise la normalisation des types et la fabrication à l'avance, en atelier, de leurs éléments. Sport, Eau et Soleil présente alors dans ses pages, un modèle de piscine-école en site terrestre de plein air ainsi qu'un bassin école flottant, celui de Neuilly-sur-Marne, construits conformément aux prescriptions du haut-commissariat à la jeunesse et aux sports et à celles de la fédération nationale. Ce type de bassin flottant, utilisable en rivière, lac ou mer, est déjà installé dans plus de cent cinquante sites en France ${ }^{19}$. Il est formé de pontons rectangulaires en tôles d'acier soudées. Une des faces du ponton, sur laquelle est fixé le caillebotis en bois, est amovible. Sur chacun de leurs cotés, les pontons présentent des ferrures spéciales d'accrochage : ils peuvent être réunis les uns aux autres dans quatre directions et former un bassin de la forme et des dimensions désirées. Sur l'un des petits cotés du rectangle de chaque ponton se trouve un assemblage destiné à faciliter la manutention et l'amarrage aux berges et au fond. Lorsque le bassin est installé, les accessoires (échelles, garde-corps, passerelles...) peuvent être placés sur les pontons ${ }^{20}$.

Si ce type de bassin connaît un réel succès du fait de sa souplesse et de son faible prix de revient, l'entre-deux-guerres voit se créer un certain nombre d'établissement où les bassins sont construits en dur.

\section{... aux piscines maçonnées}

Ces premiers bassins réalisés en maçonnerie sont encastrés dans la berge ; ils constituent un prolongement presque naturel au quai en béton, aménagement de plus en plus fréquent du rivage. À Ris-Orangis, à Corbeil ou encore à Maisons-Alfort, les bassins sont encore des pontons flottants sur trois côtés, mais le quatrième côté est constitué du quai maçonné, construit plus ou moins au niveau de l'eau. La baignade du Banc de sable de Joinville-le-Pont n'est d'abord qu'une petite crique naturelle avant d'être aménagée : le bassin est encadré par des gradins en arc de cercle du côté du rivage, et fermé du côté de la rivière par une digue. Le premier niveau de gradin est en béton afin de résister aux crues hivernales, deux autres niveaux sont d'abord recouverts de sable puis engazonnés. Au-delà du premier bain, des bouées délimitent un second bain pour les nageurs expérimentés, directement dans la rivière ${ }^{21}$.

Des préoccupations liées à la sécurité mais aussi des questions d'hygiène et de pollution justifient la tendance à isoler de plus en plus souvent le bassin du lit du cours d'eau. Même si Sport, Eau et Soleil ne mentionne ces problèmes qu'au sujet des plages de bord de mer souillées par le mazout, les conditions sanitaires des baignades en rivière sont abordées dès le XIX ${ }^{e}$ siècle. Les bains Deligny, fleuron du genre et alors point de rencontre de la haute société, sont situés après la traversée de Paris. Déjà, en 1844, Eugène Briffault remarquait que « l'école de Deligny s'est faite opulente et magnifique, mais la moindre pluie, la moindre indisposition atmosphérique rend quelquefois ses bassins vaseux. Il est alors difficile de nager longtemps dans ces eaux, sans avoir la poitrine toute souillée d'un dépôt boueux et les oreilles embarrassées ${ }^{22}$. Il faut attendre 1919 pour qu'un système de filtrage soit installé à Deligny (et même 1953, au Bain-Royal23 ${ }^{23}$, quai du Louvre). Durant l'entre-deux-guerres, à Paris, la question devient plus pressante. Le syndicat des piscines modernes et plages artificielles, dans un document d'information de 1935, évoque la question des différents systèmes d'épuration et de renouvellement de l'eau : tandis que les établissements les plus anciens comportent seulement un filtrage mécanique à travers des couches de sable fin qui retiennent les déchets et débris organiques, les 
établissements plus modernes sont dotés d'un système de stérilisation de l'eau soit par le chlore gazeux, soit par l'eau de Javel du commerce, soit par des dérivés tels que la Chloramine. Cependant, cette dernière méthode présente l'inconvénient de produire une eau au goût désagréable et irritante pour les muqueuses et les yeux. Le syndicat indique qu'il a acquis l'exclusivité d'un brevet mis au point en Hollande, source de progrès depuis plusieurs années déjà, et qui exclut l'emploi de tout produit chimique. Le procédé repose sur l'action destructive de certaines classes de bactéries sélectionnées, intensifiée par une oxygénation énergique ${ }^{24}$. Pourtant, encore en 1936, la directive officielle dispose que les établissements de bains froids doivent seulement être dératisés régulièrement ${ }^{25}$. En 1939, Jacques Meuley, étudiant en médecine lui-même adepte des bains dans la Marne, étudie dans sa thèse une pathologie qu'il attribue à la baignade en rivière, raison pour laquelle il exprime le souhait de voir de nouvelles dispositions se généraliser ${ }^{26}$. Il explique que l'eau de la Seine ne devrait plus être utilisée directement, car sa souillure est bien connue. Il prône la réalisation de bassins clos avec filtration d' « eauchloration » et renouvellement constant, tandis que les plages en rivière devraient selon lui être "ratproof » en étant installées loin des déversements d'égouts et dotées de grilles pour empêcher les animaux d'accéder à la rivière. de la baignade en rivière, ainsi la Plage de Villennes, qui ouvre en $1935^{27}$ (ill. 4).

\section{4 : Coupe longitudinale des bains flottants de Levallois-Perret}

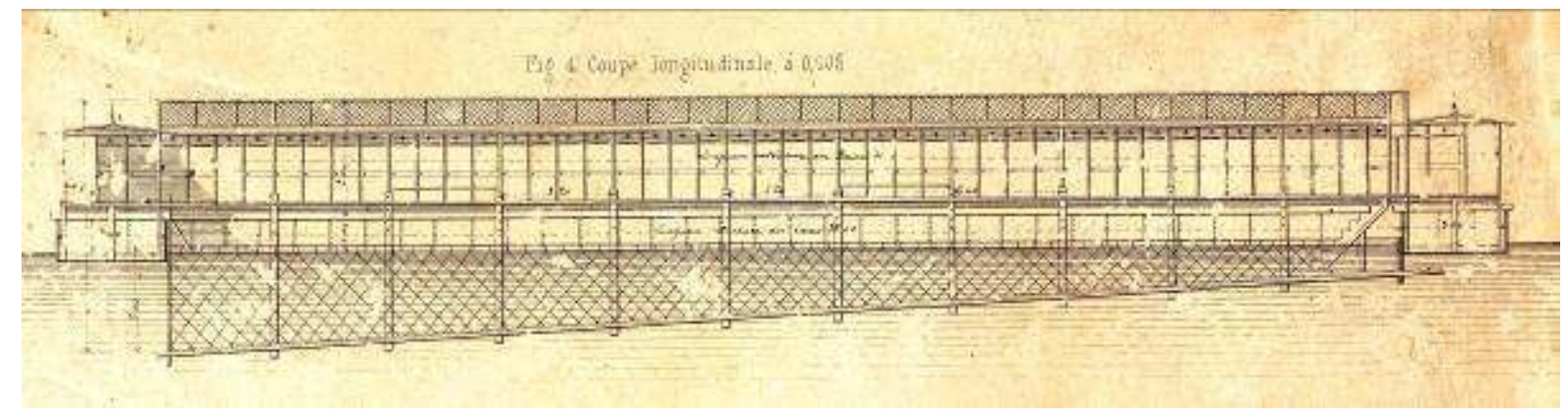

Dans Nouvelles annales de la construction, Bains et lavoirs publics, $n^{\circ} 18$, septembre 1873, planche 42

(c) musée de la batellerie, Conflans-Sainte-Honorine.

Dès 1850, les amateurs fréquentent la plage naturelle de l'Isle-Adam sur un bras de l'Oise, mais il faut attendre 1910 pour qu'un investisseur construise les premiers aménagements à l'origine de l'immense succès de l'établissement, première plage fluviale de France par sa taille, près de trois hectares. Après la Seconde Guerre mondiale, à la suite de l'interdiction de se baigner dans l'Oise, de notables améliorations sont apportées. L'eau est traitée, les bassins de filtration par le sable, à ciel ouvert, étant dissimulés par les bâtiments de cabines; le petit bassin est cimenté; le grand bassin, auparavant directement dans la rivière, est remplacé par une structure en résine, encastrée au milieu de la plage et longeant seulement la berge (un pédiluve la ceinture qui évite qu'elle ne soit souillée pas le sable). Enfin, Johnny Weissmuller, l'ancien champion de natation, alias Tarzan, inaugure le «bassin-record» en 1949. Celui-ci n'est pas enterré, mais construit hors-sol. Des tribunes entourent la cuve, à l'exception d'un des deux petits côtés où est placé le plongeoir. Les élévations extérieures sont ornées de faux colombage, eux aussi en béton, qui rappellent l'architecture des cabines ${ }^{28}$. La piscine de Viry-Chatillon présente 
quant à elle une configuration tout aussi exceptionnelle: son bassin rectangulaire s'implante perpendiculairement au rivage et seul un petit côté, fermé par une digue, borde visuellement l'eau vive, dominé par un imposant plongeoir.

\section{Autour du bassin}

Le plongeoir, aujourd'hui intrinsèquement lié au bassin de natation, en devient un accessoire incontournable dès le milieu du XIX ${ }^{e}$ siècle. Le plongeoir des bains Deligny n'échappe pas à la description des commentateurs. Constructions similaires aux premiers bassins flottants en bois, leur précarité explique que seules les images anciennes en conservent le souvenir. Ceux qui dominent les enceintes délimitées par de simples bouées sont les plus spectaculaires : la profondeur du lit de la rivière autorise les exploits. Les tentatives de record peuvent même être l'occasion d'assembler des structures éphémères, comme lors des championnats du monde de natation à Joinville en 1906 ou les championnats de France organisés par les Pingouins de la Marne au Perreux en 1922, lors du «Plongeon de la mort [en vélo !] exécuté par Vaissade le 28 juillet 1907 » ou encore lors du record du monde à $31,50 \mathrm{~m}$. de Peyrusson, toujours à Joinville ${ }^{29}$. Pour le plus grand nombre, les tremplins sont avant tout des accessoires ludiques et les propriétaires de baignade cherchent à multiplier ce type d'éléments afin de satisfaire leur clientèle. Pour les architectes qui dessinent ces plages à partir des années 1920, ils sont l'occasion de concevoir des structures élancées qui fonctionnent comme des signaux dans le paysage. Dès 1921, l'Isle-Adam possède un grand toboggan où les nageurs glissent et tombent directement dans la rivière, comme à la Plage de Villennes quinze ans plus tard ou à celle de Beaumont-sur-Oise. À la plage des Lys-Chantilly (sur la commune de Boran-sur-Oise), les baigneurs sont précipités dans l'eau dans des petits chariots dévalant une rampe sur des roulettes, très similaires à un manège. Dans ce dernier établissement, un système de vagues artificielles est installé dans le bassin et les plus aventuriers peuvent même s'exercer en pleine rivière à l'aquaplane, ancêtre du ski nautique ${ }^{30}$. À Neuilly-sur-Marne ou à Corbeil, les enfants essaient de marcher sur une roue à aubes qui les précipite dans l'eau au moindre écart d'équilibre.

Si ces jeux d'eau restent gravés dans le souvenir des jeunes baigneurs qui les pratiquèrent, ce sont davantage les bâtiments annexes construits le long des plages qui suscitent aujourd'hui l'intérêt. Bien qu'édifiés pour durer, les ensembles construits à partir des années 1920 ont quasiment tous fermé. Ils ont disparu l'un après l'autre avant que leur valeur patrimoniale ne puisse être reconnue. Ces équipements rappellent l'architecture "paquebot » en vogue durant l'entre-deux-guerres, notamment pour tous les équipements sportifs liés à l'eau ${ }^{31}$. Hublots, bastingages et mats à haubans garnis de flammes triangulaires garnissent les bâtiments tout en béton, peint d'un blanc immaculé, qui s'étirent le long du quai. Généralement, un corps principal accueille guichet, buvette, restaurant, solarium et autre boutique, tandis que les cabines et sanitaires se succèdent dans de longues ailes, plus basses. Ainsi les installations de la Plage de Champigny, réalisées suivant les plans dressés par Fromonot, architecte parisien, ouvrent en 1933. Une étendue recouverte de sable (aujourd'hui d'herbe) longe la rive ; elle est encadrée de deux bassins en béton placés dans le lit de la rivière et précédée d'un long bâtiment de cabines côté quai. Cette dernière construction est surélevée afin de se trouver protégée des crues de la rivière ${ }^{32}$. L'année suivante, ouvre la plage de Meaux-Trilport dont bientôt les bâtiments, notamment ceux du Cercle nautique, s'élèvent le long de l'étendue de 
sable, face aux bassins flottants formés de pontons. En 1935, les architectes de Poissy, Lucien et Paul-Edmond Bourgeois, réalisent la spectaculaire plage de Villennes. Citons également la plage de Gournay (le Petit-Deauville), la baignade Sainte-Catherine à Créteil, la plage municipale de Maisons-Alfort, ainsi que la plage de Lys-Chantilly où une grande rotonde aux terrasses étagées était couronnée d'un mat spectaculaire. Les bâtiments de la plage de Melun, dessinés en 1942 par l'architecte départemental Maxime Verdeaux ${ }^{33}$, et surtout ceux de l'Isle-Adam, plus anciens, se rattachent davantage à la mode néonormande que l'on retrouve également dans l'architecture sportive ${ }^{34}$. Hautes toitures étagées, toits débordants, aisseliers ouvragés, fenêtres à petits bois, faux colombages renvoient ici au pittoresque néo-régionaliste. Le restaurant de la plage est d'ailleurs baptisé «le Pavillon normand». La baignade d'élisabethville (sur les communes d'Aubergenville et d'Épône dans les Yvelines), surnommée Paris-Plage ou, elle aussi, le Petit-Deauville, ainsi que son luxueux hôtel complètent un lotissement de villégiature. La reine Élisabeth de Belgique réside dans la commune durant la guerre 1914-1918. Dès le début des années 1920, les actionnaires de la Belgique prévoyante et de la Prévoyance mutuelle créent un ensemble pavillonnaire, parrainé par la souveraine. La cité-jardin, conçue sur un plan radioconcentrique, rayonne autour de la gare de chemin de fer ${ }^{35}$. La plage et son bassin en bois flottant ouvrent en 1929. Un embarcadère permet des excursions sur la Seine en vedette ou en voilier, les touristes peuvent également profiter du casino ou du golf. Dans les années 1950, lorsqu'on ne peut plus se baigner dans la Seine, l'usine Renault de Flins, implantée tout à côté, finance la construction d'une piscine artificielle dont le bassin est encastré sur le quai. L'hôtel est reconvertit avant d'être abandonné puis squatté jusqu'à la destruction récente des vestiges encore existants 36 .

Le dimanche, des trains spéciaux, souvent directs depuis Paris, desservent ces établissements à la renommée grandissante. Après la seconde guerre mondiale, face au développement de l'automobile, les gestionnaires tentent d'aménager des parkings au plus près de leurs installations. Les Parisiens, autrefois cantonnés aux établissements installés au cœur de la capitale, ont désormais la possibilité de chercher de plus en plus loin leur baignade idéale.

\section{Nager au fil de l'eau}

\section{À Paris ${ }^{37}$}

Depuis fort longtemps, les berges du fleuve parisien sont encombrées : elles se partagent entre bateaux à lessive, passages d'eau (on en dénombre neuf en 1788) qui pallient l'encombrement des ponts, porteurs d'eau, pécheurs professionnels ou occasionnels, activités portuaires, bateaux moulin, marchés flottants... et bientôt établissements de bains. Ainsi, dès le XVII ${ }^{e}$ siècle, une réglementation organise le fonctionnement de ceuxci; en 1688, les époux Villain obtiennent l'autorisation d'installer des bains au pont Marie. Ces premiers établissements demeurent très rudimentaires. Au cours du XVIII ${ }^{e}$ siècle, ils se multiplient le long des rivages parisiens, certains mis en place uniquement pour la saison d'été (du $1^{\mathrm{er}}$ mai au 30 septembre). Ils sont démontés à l'automne; chaque ponton est remorqué pour l'hiver et abrité en dehors de Paris (à l'île des Cygnes au BasMeudon ou dans le canal de l'Ourcq par exemple). Les principaux bains sont situés à la Rapée, près de l'Archevêché ${ }^{38}$ et plus généralement aux pointes orientales de l'île de la 
Cité et de l'île Saint-Louis, quai de Morfondus (aujourd'hui quai de l'Horloge), de part et d'autre du pont Neuf, square du Vert-Galant, au port Saint-Nicolas ${ }^{39}$ ainsi que rive gauche, au port de la Grenouillère ${ }^{40}$ et à la barrière des Invalides ${ }^{41}$. Le sieur Poitevin installe les premiers bains chauds parisiens en $1757^{42}$, amarrés près du pont Royal, puis d'autres aux Tuileries, au pont Neuf et enfin au pont de la Tournelle ; son affaire est bientôt rachetée par Viguier. Les établissements proposant une eau chauffée sont rares, l'eau alimentant les bains demeurant le plus souvent à sa température naturelle, assez fraîche même durant l'été. Les bains publics regagnent leurs lettres de noblesse : le sujet est donné en concours aux étudiants en architecture de l'École des beaux-arts ${ }^{43}$. À la fin du XVIII siècle, les établissements parisiens proposent souvent des bains complets, c'est-à-dire comprenant douches, bains, bains de vapeur et bassin collectif. Turquin, propriétaire de plusieurs d'entre eux, semble inventer le concept d'école de natation qui devient un nouveau lieu de sociabilité, stimulant l'imagination des auteurs ${ }^{44}$.

En 1805, dix-neuf bains froids dont deux assignés aux juifs, quatre bains chauds appartenant au sieur Viguier et deux écoles de natation sont installés à demeure ${ }^{45}$. Depuis le XVIII ${ }^{e}$ siècle, les autorités rejettent nombre de demande d'autorisation d'installation de bains car la priorité est donnée à la navigation et le trafic augmente sans cesse. Lorsque des autorisations sont accordées, elles précisent d'ailleurs que les bains pourront être déplacés sans indemnité à la moindre injonction. Pourtant les écoles restent nombreuses parce qu'il faut répondre au besoin grandissant de la population et les ordonnances du préfet de police réitèrent chaque année l'interdiction « de se baigner en rivière dans Paris, si ce n'est dans les bains ou écoles de natation autorisés ». Cette interdiction vaut également pour le canal Saint-Martin, le bassin de la Villette, le canal de Saint-Denis et celui de l'Ourcq, dans le département de la Seine ${ }^{46}$. Seuls quelques mariniers et les établissements structurés ont l'autorisation d'organiser des baignades en pleine eau (depuis un bateau, le bachot que toute école de natation doit obligatoirement posséder pour des raisons de sécurité) qui se déroulent sept à huit fois par jour : « La pleine eau, c'est le dernier enseignement de la natation ; c'est l'essai que l'on va faire de ses forces au dehors de l'enceinte du gymnase. » Les nageurs déjà expérimentés, encadrés d'un maître de nage, remontent le fleuve dans une embarcation, plongent et suivent la barque descendant le courant en évoluant au milieu de la rivière, faisant « route avec des charognes flottantes et mille autres agréments semblables. Enfin [...] on remonte dans le bateau, qui ramène à l'école la cargaison vivante $»^{47}$. À partir de 1877 , devant l'augmentation du trafic fluvial (transport de marchandises mais aussi trafic de voyageurs), aucune nouvelle autorisation d'installation des bains sur la Seine n'est plus finalement accordée. Les bains endommagés ou très anciens ne peuvent plus être réparés ni remplacés et doivent être définitivement supprimés. Toutefois devant la pression des exploitants et en l'absence de solution de substitution, les bateaux bains et les écoles de natation se révèlent plus difficiles à supprimer que les bateaux lavoirs. Le nombre des installations permanentes demeure quasiment constant durant le siècle : dix-neuf en 1833, vingt en 1875 et encore quatorze en 1910 (ill. 5). 


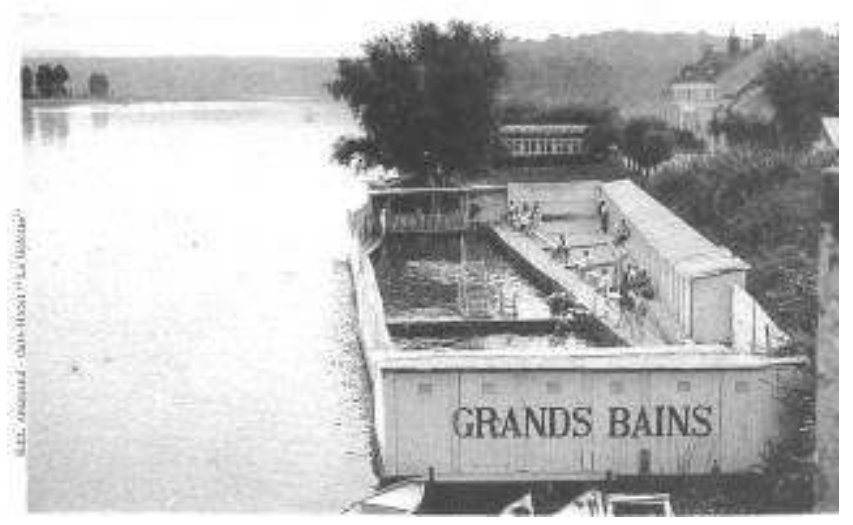

Carte postale, coll. part.

(c) Inventaire général, repro. Stéphane Asseline.

\section{Le long des rivages franciliens}

Si les premiers bains organisés sont tout d'abord installés dans Paris, ils se développent également au bord de tous les rivages de la région car la baignade en rivière est bientôt interdite en dehors des établissements de bains et de quelques lieux listés par les autorités. Ainsi en 1888, dans le département de la Seine, ne restent accessibles que quarante mètres en aval du barrage de Port-à-l'Anglais à Alfortville, un site à Vitry, cinquante mètres à l'abreuvoir de Puteaux (mais après 9 heures du soir), un autre site à Nanterre, un vis-à-vis de l'île de Chatou-Carrière et en Marne, un site à Nogent, un autre à Saint-Maur au lieu-dit « le Banc de Sable », un rivage à Champigny en amont de l'île du Martin-Pêcheur, un autre à Bonneuil en aval de l'île du Moulin-Bateau sur quatre-vingt mètres, et enfin un dernier site à Créteil dans le bras du Chapitre. Les services d'archives, grâce aux autorisations nécessaires, conservent la trace de nombre d'installations et les collections de cartes postales anciennes nous en fournissent une iconographie précieuse : les rivages de la Seine, en amont comme en aval de la capitale, de la Marne, de l'Oise, du Loing voient se multiplier les bains froids, les baignades et les écoles de natation plus ou moins élaborées ${ }^{48}$ (ill. 6). 


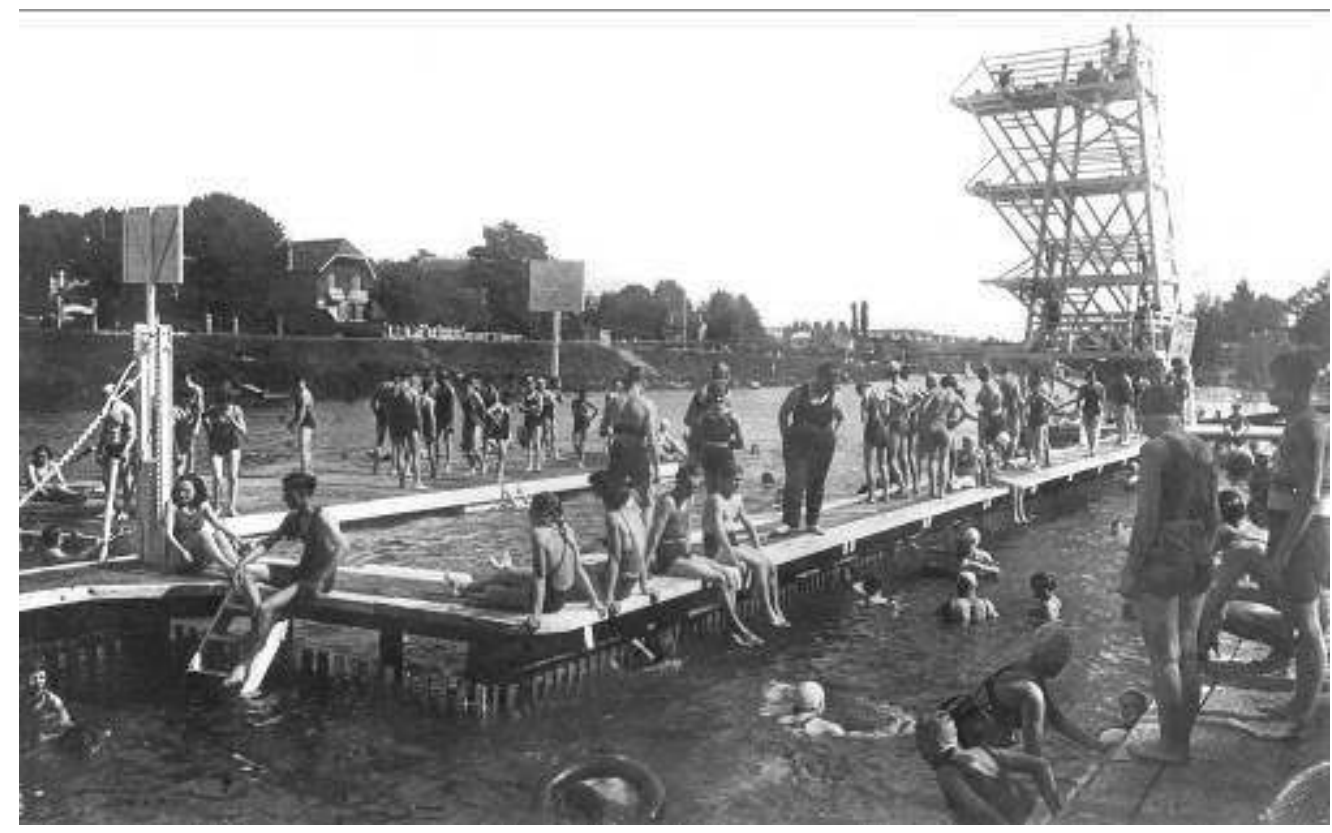

Carte postale, coll. part.

(c) Inventaire général, repro. Stéphane Asseline

29 À Melun par exemple, dans les années 1840, le sieur Bertrand obtient l'autorisation de réinstaller chaque année un établissement de bains froids destinés aux femmes, composés de toiles tendues et de pieux enfoncés dans la rivière. Un peu plus loin, l'établissement de M. Doyen est entouré de bateaux (qui accueillent les cabinets des baigneurs) qui supportent un plancher où prennent place les personnes qui souhaitent se baigner. Des piquets et des cordes à l'intérieur du bassin sont disposés de manière à empêcher que l'on puisse s'engager sous les bateaux. Le bassin, dragué, dispose d'un fond de cinquante centimètres à deux mètres au plus en pente continue. Les bains sont entièrement recouverts de toile flottante, occultant ainsi la vue depuis le rivage ${ }^{49}$. Ce type d'établissement se rencontre à Mantes, Meulan, Lagny-sur-Marne, Valvins, Choisy-le-Roi, Argenteuil, Saint-Denis, etc.

La plupart des équipements sont des entreprises simplement commerciales tirant bénéfice du nouvel engouement pour le sport et la baignade, telle la plage d'Élisabethville, celle de Villennes ou encore la plage de l'Isle-Adam (l'une des rares qui n'ait jamais cessé son activité). D'autres projets n'aboutissent pas, comme le complexe du Sport international, projeté en 1869 sur l'île Saint-Germain à Issy-les-Moulineaux et qui aurait compris deux écoles de natation, l'une pour les femmes, l'autre pour les hommes, sa trop vaste ampleur et le flou de son programme effrayant sans doute les investisseurs potentiels ${ }^{50}$; c'est également le cas du projet datant de 1893, composé de bains, lavoir et hydrothérapie, prévu sur la Seine à Draveil et dont on conserve de très beaux dessins signés de l'architecte Louis Ducastel ${ }^{51}$. Quelques équipements sont réalisés à l'initiative d'associations sportives, ainsi le bassin de l'USM (union des sauveteurs de la marne) à Nogent-sur-Marne ${ }^{52}$ ou ceux de la société d'éducation physique alsacienne-et-lorraine ${ }^{53}$ et de la société de natation et de sauvetage des pingouins de la Marne au Perreux-sur-Marne 54 (ill. 7). 


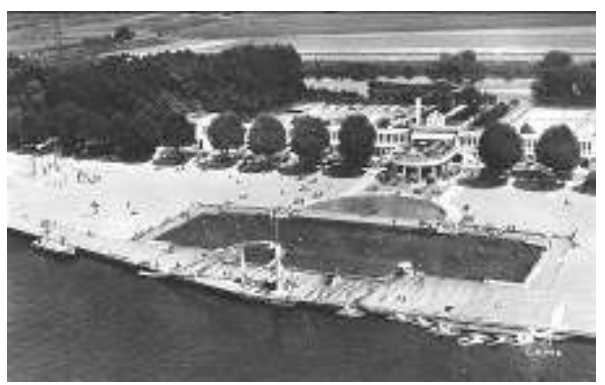

Carte postale, coll. part.

(c) Inventaire général, repro. Jean-Bernard Vialles

31 Plus récemment, afin d'encadrer les baignades sauvages et de développer la pratique sportive, certaines municipalités créent leurs propres équipements ou en reprennent d'anciens à leur compte, comme à Saint-Maurice en $1922^{55}$ ou Neuilly-sur-Marne en $1937^{56}$ . D'autres communes, faute du financement disponible pour construire un établissement municipal ${ }^{57}$, accordent des concessions sur leur rivage. C'est le cas de la plage de MaisonsAlfort-Charentonneau qui ouvre en 1930 (dont le bassin, en partie encastré sur la berge, constitue l'un des derniers vestiges encore existant des baignades en Marne) ${ }^{58}$ ou de celle de Champigny, en 1933. Ici, malgré l'interdiction préfectorale, on se baignait dans la rivière en maints endroits, pourvus d'équipements plus ou moins précaires, voire carrément sauvages (comme au pied du pont de Champigny) ${ }^{59}$. Aussi, le conseil municipal autorise-t-il l'installation d'une plage, en raison de l'intérêt de tous « au triple point de vue de l'hygiène, du sport et de la sécurité ». Il négocie avec le concessionnaire des tarifs particuliers pour les Campinois et la gratuité à certains horaires pour les enfants et les sociétés sportives locales. Cas de figure exceptionnel, le bain du Banc de sable à JoinvilleLe-Pont est construit en 1930 pour les ouvriers de l'usine Pathé-Natan et ne devient un établissement municipal que quelques années plus tard (avant d'être finalement mis en concession par la commune) ${ }^{60}$.

Au début des années 1960, les plages de rivières franciliennes sont encore très prisées. I.L. Prevost, PDG de la SA Plage de Meaux-Trilport, devient le tout nouveau président du Syndicat national des exploitants de bains de rivière. Cette organisation a pris le relais en 1961 du Syndicat des plages, piscines et baignades de France, fondé en 1935, sur l'initiative de plusieurs propriétaires de bains de la région parisienne, soucieux d'assurer la défense de leurs intérêts ${ }^{61}$. Le syndicat est affilié à la fédération des syndicats d'exploitants des piscines, patinoires et établissements de bains de mer et de rivière, fondée la même année et qui dispose de sa revue Sport, Eau et Soleil, pour soutenir la profession. Les membres y exposent leurs revendications: la réglementation sur les débits de boissons alcoolisées les pénalise; le régime juridique fixant leurs conditions d'occupation du domaine public maritime ou fluvial les maintient dans une grande précarité qui interdit les investissements importants; ils s'élèvent contre l'arbitraire administratif qui augmente sans justification leur redevance pour l'occupation du domaine public; en outre, l'administration des domaines aurait tendance selon eux à renoncer aux contrats de gré à gré pour l'attribution des lots de terrains du domaine public, préférant l'adjudication publique, procédure "qui fait bon marché » des droits acquis comme des frais engagés par les concessionnaires; faute d'une convention collective, ils éprouvent les plus grandes peines à recruter des maîtres nageurs pour la 
saison estivale ; ils s'élèvent également contre les prétentions exorbitantes de la SACEM ; ils souhaitent enfin une application plus stricte des textes réglementant la circulation des bateaux à moteur. Moins de dix ans avant l'interdiction des baignades en rivière pour cause de pollution, on ne trouve pas un mot de ce sujet. Pourtant, la santé des nageurs, à l'origine des baignades en eau vive, sera aussi la raison de leur disparition.

\section{Se baigner : le corps, l'eau, le plaisir}

\section{Entre hygiène et santé}

Le bain en rivière se développe à partir du XVIII siècle car son bénéfice pour la santé est désormais acquis, il préviendrait même certaines maladies. La définition du «Bain de santé ou de propreté (en Médecine.) » de l'Encyclopédie explique que :

Les médecins toujours attentifs à chercher des secours contre les maladies, remarquèrent les bons effets qu'il produisait, et le mirent au nombre de leurs remèdes.

Willis nous en donne un exemple dans son traité de la Phrénésie ${ }^{62}$, à l'occasion d'une fille qui fut guérie de cette maladie par un seul bain froid que l'on lui fit prendre : cette malade était dans cet état depuis plusieurs jours ; les saignées, les délayants ${ }^{63}$, les amples boissons émulsionnées, etc. n'avaient pas pu diminuer la fièvre violente dont elle était attaquée, et la soif qui la dévorait. Le bain d'eau simple pris dans la rivière, pendant un quart d'heure, calma tous les accidents, lui procura un sommeil tranquille, et elle fut guérie sans avoir besoin d'autres remèdes. On trouve dans la pratique plusieurs exemples de ces guérisons miraculeuses arrivées par hasard ; car souvent des gens attaqués de frénésie se sont jetés d'eux-mêmes dans des fontaines ou bassins, et ont été guéris. Ce que l'on peut encore assurer, c'est que l'usage des bains de rivière, pendant les chaleurs de l'été, est un sûr préservatif contre les maladies qui règnent ordinairement dans cette saison ${ }^{64}$.

La terminologie évolue peu à peu; il n'y a qu'un pas du concept de simple baignade à celui de la pratique de la nage, laquelle n'est pas initialement considérée comme une activité sportive mais comme un principe d'hygiène corporelle. «La natation a toujours été l'un des amusements de la jeunesse; il est de plus un des exercices les plus utiles pour la santé. Aucun autre ne favorise au même degré le développement des muscles et l'extension de la poitrine " précise Larousse en 1866. Cette nouvelle hygiène corporelle est même prescrite pour les femmes :

Depuis quelques années, il se fait dans les grandes villes, et surtout à Paris, une révolution remarquable dans les habitudes des femmes. Autrefois, on comptait à peine quelques établissements de bains de rivière qui leur fussent destinés ; et encore ces bains avaientils une fort mauvaise réputation. Maintenant, les personnes auxquelles leur position, leurs occupations ou des liens de famille interdisent les voyages et les bains de mer, trouvent des établissements fort bien tenus, où l'on veille tout à la fois au confortable, à la sécurité et à la décence. La mode a pris les bains froids sous son patronage, et, cette fois au moins, elle se trouve d'accord avec les lois de l'hygiène. Chaque été [...] les femmes [...] qui restent prisonnières dans nos villes se rendent aux écoles de natation. [...] La plupart des femmes, à Paris surtout, abusent des bains chauds. Ces bains sont pris trop souvent à cette température, ramollissent beaucoup les chairs et leur donnent une blancheur blafarde. Les bains de rivière et surtout ceux de mer peuvent très bien porter remède à ces inconvénients. 

l'élément liquide sont constamment condamnés et la puissance publique ne cesse, au cours des siècles, de réglementer les baignades afin d'éviter les infractions aux bonnes mœurs. La réputation de lieu de prostitution s'attache même à certains établissements de bain. Non seulement les baignades seraient des lieux propices à la licence mais la seule vue des baigneurs heurte les passants. À partir du XVIII ${ }^{e}$ siècle, dans les ordonnances parisiennes régulièrement renouvelées, un article précise qu'aucune communication ne peut être établie entre les bains d'hommes et ceux de femmes ; ils doivent être séparés, éloignés les uns des autres et les bassins occultés. En 1777, le lieutenant de police évoque l'occasion de libertinage que fournit la baignade dans la Seine, ce qui suscite un sujet de scandale, des plaintes de riverains, qui se disent obligés d'installer les enfants dans les chambres reculées pour les soustraire à ce spectacle indécent et contraire aux bonnes mœurs. L'ordonnance de 1783 confirme l'interdiction de la baignade en plein jour en Seine, ne la tolérant qu'en amont ou en aval de la ville, ainsi que la nuit. clientèle aisée), les hommes portent «un pagne génital» et les femmes une chemise. Malgré tout, ces dernières préfèrent la partie du bateau du côté du quai, davantage à l'abri des regards indiscrets. Peu à peu, le costume de bain se spécifie et quelques années plus tard, dans les écoles de natation, «les baigneuses, vêtues de laine foncée noire ou brune, n'ont de nu que le col, les pieds et les bras; le pantalon-caleçon est à plis, en blouse, afin qu'il ne puisse pas coller sur les formes; presque toutes les femmes portent un serre-tête $»^{66}$. L'atmosphère semble tout opposée dans les bains à quatre sous ${ }^{67}$ :

La nature, en ces lieux, vigoureuse et sauvage,

D’un plaisir frelaté ne connaît point l'usage ; 
Mais la santé robuste, et la mâle vigueur,

La bruyante gaîté, coloris du bonheur,

Répand sur ce tumulte et la joie et la vie.

L'eau tiède et captive, en ces bains, est bannie,

Et la Seine, en son cours fier de sa liberté,

Fait rouler dans ses flots la force et la santé.

Tel on voit un essaim de fraîches Néréides,

Ondulant leurs contours sur les plaines liquides,

La grisette, sans voile, offre, nus, au soleil

Ses appas rebondis, son teint frais et vermeil.

Dans cette catégorie d'établissement :

composé de quelques pieux enfoncés dans le sable, auxquels sont mal ajustés des planches grossières qui forment la ceinture [...], une toile à voiles interdit aux Actéons modernes la vue de toutes nos Dianes au bain: non pas que beaucoup en fussent formalisées, et qu'aucune même voulut changer les curieux en cerfs ; mais c'est ici, à défaut de pudeur, une mesure de police; et dans l'absence de la vertu, on impose un règlement de décence. On voit là pèle mêle des filles de tous âges se baignant totalement nues toutes ensembles. Les vêtements sont mis par paquets et fixés à des cerceaux qui soutiennent la toile en demi-cintre ${ }^{68}$.

En fait, dans les bains à quatre sous, les troubles les plus fréquemment mentionnés sont les disputes de poissardes ou les plongeurs intrépides qui se glissent entre deux eaux parmi les baigneuses.

L'auteur, Cuisin, mentionne également l'existence de bains à six sous destinés à une population moins modeste mais «alors, vous êtes seul entre quatre planches, comme un fou dans son cabanon ». Malgré cet isolement, la licence n'est pas l'apanage de la classe populaire, en témoigne le séjour, certes vénitien, durant l'été 1865, de la comtesse Livia pendant lequel le lieutenant Remigio devient son amant, au bain flottant de Rima ${ }^{69}$.

Durant tout le XIX ${ }^{e}$ siècle, les mêmes interdits sont reconduits d'année en année, allant jusqu'à s'appliquer aux enfants, comme à Noisy-le-Grand en 1888. Dans son règlement des baignades, le conseil municipal dispose qu'il leur est interdit de se baigner sans caleçon ${ }^{70}$. La baignade de pleine eau est également rigoureusement encadrée : les bachots des mariniers doivent être surmontés d'une tente quand les baigneurs se déshabillent à bord ${ }^{71}$. Le poème de Raymond Radiguet, écrit vers 1918 et évoquant la Marne, atteste du caractère implicitement érotique de la baignade en rivière. Le jeune prodige a vécu l'essentiel de sa trop brève vie à Saint-Maur-des-Fossés, à deux pas de la rivière :

Le rendez-vous solitaire

Emprunte aux oiseaux leur berge

Au feuillage d'ardoise tendre!

Loin des fatigues, ma cycliste,

Qui t'épanouis sur nos berges,

Future fleur comme Narcisse,

Tu sembles toi-même t'attendre! 
Durant l'entre-deux-guerres, la nudité, même partielle, reste choquante et continue d'être proscrite dans l'espace public. Ainsi, le règlement sanitaire de la Ville de Paris de 1936, dans son article relatif aux bains froids, précise que les établissements doivent être couverts de telle sorte que des quais, des ponts ou des habitations riveraines, la vue ne puisse plonger à l'intérieur. Les établissements implantés en ville, placés à l'abri des regards grâce à un rideau d'arbres et proposant une baignade totalement découverte, n'en ont que plus de succès. Toujours dans les années 1930, à Villennes-sur-Seine, afin d'éviter tout problème devant l'incompréhension qu'elle suscite, la communauté naturiste de Physiopolis indique dans son règlement qu'«en bordure de Seine, à l'embarcadère, ainsi que pour les baignades, les hommes doivent porter des culottes courtes arrivant à la naissance des cuisses et les femmes des maillots de bain complets »; les slips pour les hommes et les maillots deux pièces pour les femmes sont interdits ${ }^{73}$.

78 À partir du milieu du XX $\mathrm{X}^{\mathrm{e}}$ siècle, la libération des mœurs, le développement de la natation comme pratique sportive et des différents loisirs aquatiques au cœur des villes, expliquent l'assouplissement des règlements : désormais, la vue d'un nageur en costume de bain ne choque plus le citadin; la piscine constitue un lieu de flirt, désormais parmi tant d'autres.

\section{Du sport aux loisirs}

79 «Si le bain en baignoire est salutaire, combien préférable est le bain en piscine, où l'on n'a pas besoin de garder l'immobilité, et dont l'exercice de la natation vient décupler les bons effets » expose en 1886 Paul Christmann, président de la société des gymnases nautiques ${ }^{74}$. Dans son ouvrage, il explique la nécessité pour tout homme de savoir nager et vante la natation comme pratique sportive à part entière. Pour cela, il estime nécessaire de bâtir des établissements un peu partout, à commencer par Paris, ville largement sous-équipée si on la compare à Londres ou à d'autres grandes villes étrangères. Il se trouve d'ailleurs à l'initiative de la construction de la première piscine parisienne totalement artificielle : la piscine de Château-Landon qui ouvre en 1884. Par ailleurs, il indique que les baignades en rivière, activité largement saisonnière, ne peuvent pas répondre au besoin d'un entraînement régulier et rigoureux. L'encyclopédie des sports, en 1924, précise :

Il nous faudra pendant de longues années encore nous contenter des seules baignades en mer ou en rivières [...] De ce côté même, la situation de la France est encore loin d'être des

Livraisons de l'histoire de l'architecture, 14 | 2007 
plus brillantes car ces installations sont le plus souvent rudimentaires et condamnent, en même temps que toute pratique hygiénique, toute pratique sportive. Il n'est pas en effet de natation sportive, pas d'enseignement même théorique et naturellement de progrès possible dans des bassins mal délimités, au courant naturellement irrégulier, quelquefois violent, circonstances matérielles qui empêcheront tout renseignement précis, tout contrôle de l'effort et de la performance ${ }^{75}$.

Effectivement, les bassins en rivière destinés à la natation sportive, à l'instar de celui de l'Institut national des sports à Nogent-sur-Marne, constituent-ils une exception. Encore le contexte est-il spécifique puisque ce bassin succède, dans l'entre-deux-guerres, à un précédent équipement plus sommaire, réalisé pour l'École normale militaire de gymnastique (fondée en 1852 et devenue le bataillon de Joinville) dans laquelle les soldats s'exerçaient depuis la fin du XIX'e siècle ${ }^{76}$ (ill. 8).

\section{8 : Le bassin et son plongeoir de l'institut national des sports à Nogent-sur-Marne}

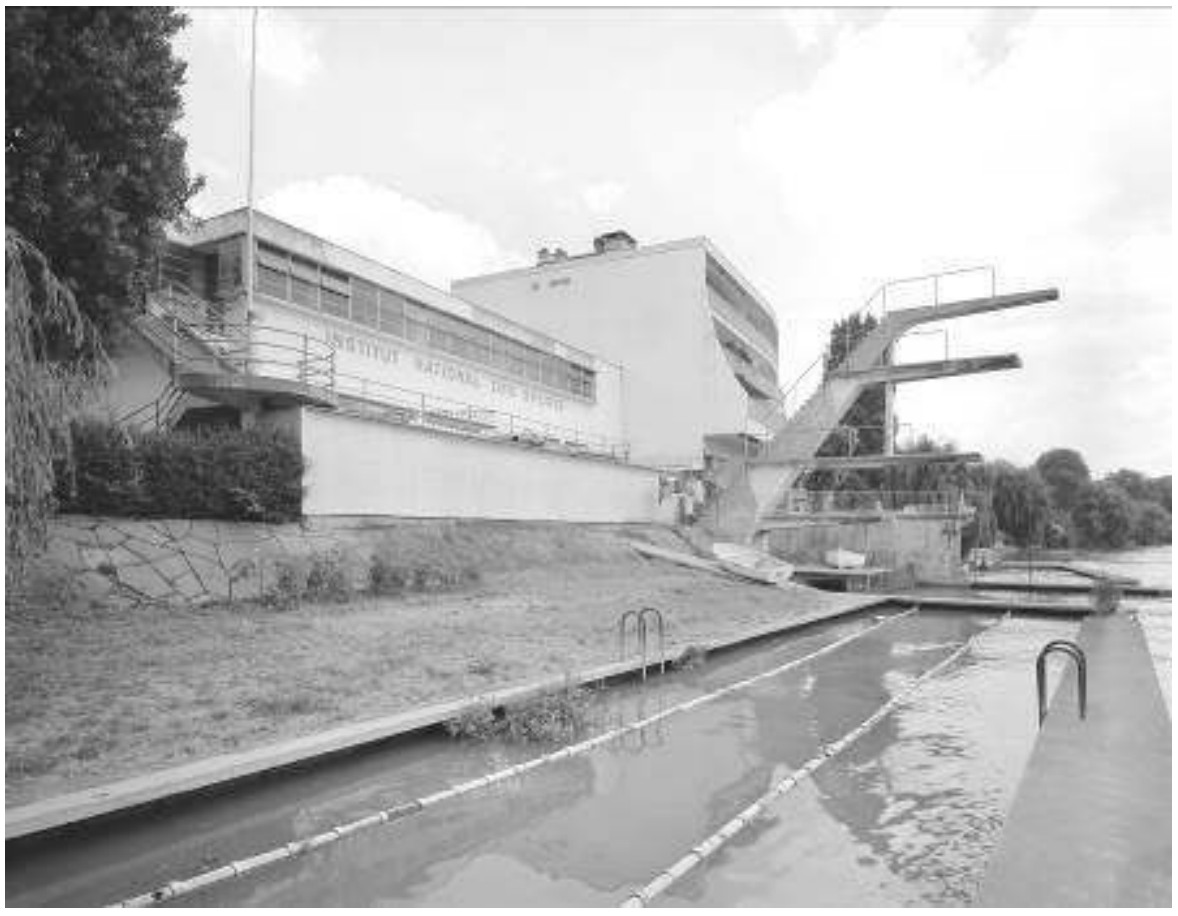

L'équipement, photographié en 1986, est aujourd'hui détruit

(c) Inventaire général, phot. Christian Décamps.

Ainsi, tandis que les piscines artificielles peinent à se construire, les bassins en rivière, longtemps les plus nombreux, conservent une double vocation. Ils offrent au plus grand nombre la possibilité d'un apprentissage encadré de la natation et se confirment comme espace de loisirs pour tous. Dès le XIX ${ }^{e}$ siècle, les bains de rivière comprennent des espaces de détente variés : ils disposent de divers aménagements autres que le bassin qui permettent aux clients d'y passer du temps et d'y dépenser de l'argent agréablement. Eugène Briffault note en 1844 qu' "une journée à l'école de natation est un des plus piquants tableaux de la vie parisienne ». À la mi-journée, « s'organisent des déjeuners que le boulevard Italien et la rue Montorgueil pourraient envier. Le bain reste désert et l'eau n'est fréquentée que par quelques gens à jeun, et ceux qui se baignent du bout des pieds, en attendant que les côtelettes soient cuites; on entend quelques explosions de bouteilles 
de vin de Champagne; le café, le gloria et le punch parfument l'atmosphère: le cigare fume partout $»$. jamais : ils viennent là comme au bal masqué " pratiquer la " conversation en déshabillé " conclut un troisième chroniqueur ${ }^{78}$. On a vu qu'aux alentours de la capitale, les baignades sont fréquemment associées à des guinguettes, des bals musette ou des locations de barques. Les bords de Marne, dans les derniers méandres de la rivière avant qu'elle ne se jette dans la Seine, concentrent sur quelques kilomètres des dizaines d'établissements qui font, dès les beaux jours, la joie des Parisiens. Bientôt, sur ces plages enfin «libres", hommes et femmes se baignent ensemble. Un bassin est spécialement aménagé pour les enfants ainsi que de nombreux jeux. Durant l'entre-deux-guerres, quelques établissements d'envergure acquièrent une telle renommée qu'il faut y être vu, pas nécessairement dans le bassin, mais aussi à la terrasse du restaurant ou le long de la promenade $^{79}$. Après guerre, face à cette demande croissante de loisirs nautiques des populations citadines et devant le coût des piscines artificielles couvertes, auparavant plutôt conçues pour la pratique sportive, les pouvoirs publics réévaluent leurs prescriptions et encouragent des ensembles comprenant plusieurs bassins aux vocations variées, dont certains peuvent être découverts. En 1963, le haut-comité des sports crée une commission «loisirs et sports de plein air » qui prescrit l'ouverture d'installations polyvalentes comprenant espace caravaning, divers équipements sportifs et bassin de natation ${ }^{80}$. C'est dans ce contexte que le district $^{81}$ inscrit au schéma directeur d'aménagement de l'île-de-France la création de bases de loisirs, pour la plupart implantées près des rivages de la Seine ou de la Marne ${ }^{82}$. Les municipalités disposant de rivages construisent des établissements artificiels, mais en plein air et au plus près de l'eau afin de conserver une certaine atmosphère; ainsi la piscine de l'île aux Dames à Mantes-la-Jolie, due à l'architecte Lemercier et donnée en exemple par le ministère de la jeunesse et des sports en $1966^{83}$ (qui ferme pourtant en 1996), ou bien encore le centre nautique de Nogent-sur-Marne qui ouvre en $1971^{84}$. Quelques entrepreneurs s'avèrent des précurseurs en la matière, comme le propriétaire de la piscine du Lido à Chennevièressur-Marne, construite en $1937^{85}$, tandis que d'autres se lancent dans l'aventure hasardeuse pour sauver leur établissement, ainsi le beach sports nautiques de la VarenneSainte-Hilaire (Saint-Maur-des-Fossés) où un bassin artificiel, implanté de l'autre côté du quai, remplace le bassin en rivière ${ }^{86}$. La piscine disparait pourtant dans les années 1990 remplacée par un ensemble immobilier.

Les bains en rivière ne sont pas une particularité de l'île-de-France, on connaît des piscines dans toute la France, tels les bains municipaux de Chalons-en-Champagne ${ }^{87}$, les piscines en bord de Seine de Vernon ou de Loire à Tours, les baignades de Bordeaux ${ }^{88}$, Lyon ou Avignon ${ }^{89}$. Cependant, l'absence de rivage marin à proximité et l'importance de la clientèle potentielle expliquent le nombre d'équipements le long des cours d'eau franciliens. Ces installations sont d'ailleurs généralement conçues selon les mêmes 
critères esthétiques que les installations balnéaires en vogue depuis le XIX ${ }^{e}$ siècle le long des côtes françaises.

À partir du milieu du XXe siècle, le développement des transhumances estivales et celui de l'automobile, les aléas du climat parisien, enfin la pollution des rivières expliquent la baisse de fréquentation des établissements, jusqu'à leur fermeture complète lorsque le couperet de l'arrêté préfectoral d'interdiction de la baignade tombe. Les vastes bases de loisirs régionales ainsi que quelques autres, initiées par d'autres collectivités territoriales, accueillent désormais les Franciliens qui souhaitent nager en eau vive ${ }^{90}$. Les échecs des tentatives de reconversion ou la terrible pression foncière entraînent la disparition des anciennes baignades fluviales et de leurs installations. Espérons que la plage de Villennes, actuellement l'une des mieux conservées, fermée il y a quelques années et tout récemment vendue, ne disparaîtra pas à son tour. Son charme et sa situation toutes particulières - implantée sur une île de la Seine non viabilisée, elle n'est accessible qu'en bateau - devrait permettre de la sauver. La transformation réussie de la plage de Champigny en base nautique, dès 1973, atteste une possible solution. Depuis 2006, celle-ci accueille la manifestation Champigny-Plage durant le mois de juillet, malheureusement sans possibilité de baignade.

Symbolique d'une pureté retrouvée, les édiles souhaitent désormais pouvoir autoriser de nouveau les baignades en rivière. On connaît la promesse de Jacques Chirac, alors maire de Paris, de se baigner à Paris dans la Seine à la fin de son mandat ou bien encore le syndicat mixte "Marne vive $~^{91}$ dont l'objectif est la restauration de la baignade en Marne. Sous l'impulsion de Paris-plage, les opérations se multiplient - même sans rivière - telle la «plage au stade de France » en juillet 2007. Durant l'été 2007, la ville de Meaux a quant à elle, réellement rouvert sa plage. Le lieu, acquis par la municipalité, a été restauré : $800 \mathrm{~m}^{2}$ de sable fin, un large espace engazonné, des pontons en bois et une zone de baignade longue de 80 mètres dans la Marne accueillent désormais les habitants durant l'étép2. Malheureusement, les bâtiments des années 1930 sont perdus. En revanche, ceux de la piscine de Boran-sur-Oise devraient voir leur rénovation commencer à la fin de l'année 2007, en vue de leur réouverture au public. Le conseil général du Val-de-Marne, dans son engagement 112 concernant l'amélioration des berges de la Seine et de la Marne, programme l'aménagement du site de la plage de Vitry-sur-Seine en 2008. Face à cette nouvelle demande, le ministère de l'écologie et du développement durable vient de modifier la législation. Un décret du 15 mai 2007 impose, pour la première fois, un recensement des eaux de baignade avant le 30 avril 2008. Ces eaux seront inscrites au registre des zones protégées dans le code de l'environnement.

D'ores et déjà, on se baigne à nouveau dans la Seine à Paris, depuis l'ouverture de la piscine Joséphine-Baker, en juillet 2006 - la dernière piscine flottante, les bains Deligny, a coulé treize ans auparavant. L'atelier Sequana Architectures (Robert de Busni, architecte) a conçu une coque en béton garnie de flotteurs acier en sous-face. L'eau d'alimentation du bassin est pompée dans le fleuve et traitée avant d'être épurée et rejetée après usage. La couverture coulissante en verre et en acier permet d'ouvrir le bassin aux beaux jours tout en le maintenant en activité en toutes saisons. Des espaces de remise en forme, hammam, sauna, jacuzzi, salle de musculation ainsi qu'une cafeteria, un solarium et un deck sur le quai complètent l'ensemble ${ }^{93}$. Dès 2006, la Ville de Paris a annoncé le lancement d'un second projet similaire, prévu dans le quinzième arrondissement, près du parc André Citroën, et qui devrait être livré en 2010. Le conseil général des Hauts-de-Seine affiche quant à lui un projet de piscine constituée d'une barge flottante, dans la future base 
nautique de lî̂le de Monsieur à Sèvres: une nouvelle page de l'histoire des piscines en rivière d'île-de-France est en cours d'écriture, qu'il s'agisse de plages fluviales ou de bassins flottants.

\section{NOTES}

1. Cette étude se fonde sur les travaux du service de l'Inventaire général du patrimoine culturel de la région Ile-de-France. J'en remercie les chercheurs Antoine Le Bas, Brigitte Blanc et Judith Förstel pour leur aide, ainsi que Marianne Castan, conservatrice adjointe au musée de la batellerie de Conflans-Sainte-Honorine et Joanne Vajda, enseignante associée à l'école nationale supérieure d'architecture de Paris-la Villette et auteur de Paris : rendez-vous cosmopolite. Du voyage élitaire à l'industrie touristique. 1855-1837, thèse EHESS, 2005.

2. . Les Bains de Paris et des principales villes des quatre parties du monde; ou le Neptune des dames, orné de jolies gravures. Description hydrographique des thermes, bains, étuves, eaux minérales et fontaines les plus célèbres du globe ; renfermant des leçons d'hygiène, précieuses pour la beauté des femmes et la santé des hommes; ainsi que des vers, des anecdotes, des tableaux instructifs et amusants, des moeurs de divers peuples ; des galanteries décentes et des folies de bon ton. Dédié au beau sexe. Par Cuisin..., Paris, Verdière, 1822, vol. 1, p. 63.

3. Commune de Vulaines-sur-Seine, à côté de Fontainebleau en Seine-et-Marne.

4. «Tout le monde, dit La Bruyère, au chapitre VII de ses Caractères, connaît cette longue levée qui borne et qui resserre le lit de la Seine du côté où elle entre à Paris avec la Marne, qu'elle vient de recevoir. Les hommes s'y baignent au pied pendant les chaleurs de la canicule ; on les voit de fort près se jeter dans l'eau, on les en voit sortir, c'est un amusement. Quand cette saison n'est pas venue, les femmes de la ville ne s'y promènent pas encore ; et quand elle est passée, elles ne s'y promènent plus. » Les satires du temps n'ont pas épargné la promenade de la porte SaintBernard. Une comédie représentée au Théâtre-Italien en 1696 a pour titre Les Bains de la porte Saint-Bernard. ». Commentaire (appel de note $\mathrm{n}^{\circ}$ 4) de la lettre datée du 9 août 1732 de Buffon à Richard de Ruffey, dans la version publiée par Henri Nadault de Buffon, Correspondance inédite de Buffon, à laquelle ont été réunies toutes les lettres publiées jusqu'à ce jour, Paris, Hachette, 1860. Dans cette lettre, Buffon indique que, durant ces chaleurs, les princesses vont voir nager les jeunes gens à la porte Saint-Bernard.

5. Françoise de Bonneville, Le Livre du bain, Paris, Flammarion, 1997 ; Paul Négrier, Les Bains à travers les âges, Paris, la Construction moderne, 1925 ; Paul Benoit, Karine Berthier, Joséphine Rouillard, «Laver et baigner son corps en Île-de-France à la fin du Moyen Âge », Paris et Île-de-France, mémoires, t. 56, 2005, p. 27-48.

6. Selon M. Carayon-Gentil, ingénieur hygiéniste à la préfecture de la Seine, Sport, eau et soleil, $\mathrm{n}^{\circ}$ 4, octobre 1963, p. 28-29.

7. Bateau à fond plat destiné au transport du fret. La remontée de l'embarcation à son lieu d'origine étant très coûteuse, arrivée à destination elle pouvait être détruite ou vendue.

8. Première édition de l'Encyclopédie, ou dictionnaire raisonné des sciences, des arts et des métiers de Diderot et d'Alembert (1751-1772). http://portail.atilf.fr/encyclopedie/index.html (projet ARTFL de l'université de Chicago et laboratoire ATILF du CNRS). 
9. À ne pas confondre avec les fameux bains chinois du boulevard des Italiens.

10. Avis au public sur l'établissement d'une école de natation. [Paris: Imprimerie polytype, 1786]. (Prospectus concernant les bains publics établis à Paris au pont de la Tournelle par le sieur Turquin). [Turquin]. Projet d'une école de natation en faveur de la garde bourgeoise nationale de Paris, [Paris], impr. de Valleyre aîné, [1790].

11. Eugène Briffault, Paris dans l'eau, Paris, J. Hetzel, 1844. p. 63.

12. Edmond Texier, Tableau de Paris, Paris, Paulin et Le Chevalier, 1852, t. 2, p. 5.

13. Bernard Toulier, « Un parfum d'Orient au cœur des villes d'eaux », In Situ, février 2006.

14. Paul Négrier, Deux siècles d'architecture sportive à Paris : piscines, gymnases, Exposition, mairie du $\mathrm{XX}^{\mathrm{e}}$ arr., mairie du XVII ${ }^{\mathrm{e}}$ arr., Paris, délégation à l'action artistique de la Ville de Paris, 1984 et Eugène Briffault. op. cit. p. 69-76.

15. Les gibasses sont des renforts placés en surépaisseur aux endroits les plus vulnérables des flancs du bateau. Voir Dictionnaire fluvial et batelier, http://projetbabel.org/fluvial/g.html.

16. Ordonnance portant règlement sanitaire de la Ville de Paris, 10 août 1936, article 52, relatif aux bains froids.

17. Thomas Deschamps, Plages en ville, baignades en Marne, Paris, éd. Johanet, 2003, p. 45.

18. Thomas Deschamps, ibid., p. 160-163.

19. Selon le rapport de François Missoffe, L'Équipement sportif et socio-éducatif en France et ses perspectives d'avenir, établi en 1966, cette première loi-programme a permis la réalisation de 445 piscines de plein air. Voir Évelyne Combeau-Mari, «Les premiers équipements sportifs à la Réunion: une politique de l'État (1956-1971)», Staps, Revue internationale des sciences du sport et de l'éducation physique, $\mathrm{n}^{\circ}$ 61, 2003.

20. Sport, Eau et Soleil, $\mathrm{n}^{\circ}$ 3, 1963.

21. Thomas Deschamps. op. cit. p. 80-81.

22. Eugène Briffault. op. cit. p. 67-68.

23. Voir sa photographie par Marcel Bovis www.culture.gouv.fr, base de données Mémoire, réf AP73L05412.

24. Note sommaire sur le développement des piscines modernes et des plages artificielles en France par le syndicat des piscines modernes et plages artificielles. Vers 1935. Sceaux, centre de documentation de l'île-de-France.

25. Règlement sanitaire de la ville de 1936, article 52, paragraphe 7.

26. Jacques Meuley, À propos de la spirochétose ictéro-hémorragique d'origine fluviale : nécessité d'une nouvelle réglementation des bains de natation en rivière, Paris, Vigot frères, 1939.

27. Située en bord de Seine sur la commune de Médan. Voir Inventaire général, Isabelle Duhau (réd.), Jean-Bernard Vialles (phot.), Autour d'Orgeval, de la boucle de Poissy au pays de Cruye, Yvelines, Paris, APPIF, 2000, (Images du patrimoine $\mathrm{n}^{\circ} 200$ ), p. 76-77 et «La plage de Villennes », La Construction moderne, 13 octobre 1935.

28. Thomas Deschamps. op. cit., p. 14-16.

29. Cartes postales. Arch. dép. Val-de-Marne, 2Fi.

30. France 3 Nord Pas-de-Calais / Picardie. Journal télévisé 12/13 H du 05 septembre 2007.

31. Inventaire général, Antoine Le Bas (réd.), Architectures du sport. Val-de-Marne - Hauts-de-Seine, Paris, Connivences, 1991. Cahiers de l'inventaire, 23.

32. Inventaire général, Isabelle Duhau (réd.), Stéphane Asseline (phot.), Bry et Champigny. Dans les méandres de la Marne, Lyon, Lieux-Dits, à paraître fin 2007. Images du patrimoine $n^{\circ} 247$, p. 82.

33. Voir le dossier d'inventaire IA77000573.

34. Inventaire général, Antoine Le Bas (réd.), Architectures du sport, op. cit.

35. En 1927/28 est également construite la chapelle du quartier, commandée à l'architecte Paul Tournon qui réalise un édifice innovant, tout en béton armé. 
36. Voir le Courrier de Mantes, 7 septembre 2000, 17 septembre 2003 et 22 février 2006. www.epone.fr. Marc Brabant et Stéphane Degoutin, "Quand le péri-urbain était un idéal ", Urbanisme, $\mathrm{n}^{\circ} 328$, janv-fév. 2003. Élisabethville est également connu pour la cité pavillonnaire construite par Bernard Zehrfuss en 1953.

37. Paul Négrier, Deux siècles d'architecture sportive à Paris, op. cit., Isabelle Backouche, La Trace du fleuve : la Seine et Paris, 1750-1850, Paris, Éd. de l'École des hautes études en sciences sociales, 2000. (Civilisations et sociétés ; 101); Sylvie Hamel, L'Espace des loisirs: les établissements balnéaires à Paris-Ile-de-France de 1850-1950, économie des loisirs, innovations techniques et reconversions, mémoire de DEA, Histoire des techniques CDHT 2002, sous la direction de Liliane Hilaire-Perez, conservatoire national des arts et métiers, 2002.

38. Situé sur l'île de la Cité, derrière Notre-Dame, jusqu'en 1831.

39. Au pied du Louvre.

40. Au niveau de l'actuel musée d'Orsay.

41. Au niveau de la rue Constantine, bordant l'esplanade des Invalides.

42. La première tentative de réaliser un bassin de natation sur la Seine avec de l'eau chauffée remonte à 1848. La construction n'aboutit pas, son entrepreneur ayant été ruiné par les événements révolutionnaires. La règle resta la température naturelle pour les baignades d'eau vive en plein air. Voir Sylvie Hamel, op. cit. p. 46.

43. Épreuves en avril 1765, pour des bains publics sur l'île aux cygnes ; en janvier 1770, pour des bains publics à la pointe de l'île Saint-Louis. Voir Jean-Marie Pérouse de Montclos, Les Prix de Rome : concours de l'Académie royale d'architecture au XVIII siècle, Paris, École nationale supérieure des beaux-arts, Berger-Levrault, 1984.

44. Louis Debuire du Buc. Les Dames à l'école de natation, chansonnette. Lille, imp. de A. Lévy, 1859 ; Charles de Livry, Adolphe de Leuven et Alphonse Signol. L'École de natation, tableau-vaudeville en 1 acte, [Paris, Variétés, 5 août 1828], Paris, J.-N. Barba, 1828.

45. Arch. nat., $\mathrm{F}^{13}$ 593, cité par Isabelle Backouche, op. cit., p. 319.

46. Eugène Briffault, op. cit., p. 128. Malgré l'interdiction de se baigner dans le canal de l'Ourcq, les cartes postales gardent la mémoire de quelques baignades «sauvages » aux Pavillons-sousBois, au Vert-Galant, à Villeparisis ou à Mitry-Mory.

47. Eugène Briffault, op. cit., p. 116.

48. Delcampe, portail multinational de ventes d'objets de collection, dont les cartes postales, représente aujourd'hui une source iconographique accessible à tout moment et d'une étonnante richesse.

49. Arch. dép. Seine-et-Marne, 3 S 118.

50. Y étaient également prévus une école de gymnastique, un centre d'hydrothérapie, une école d'équitation, une piste pour les courses de chevaux, une tribune des joutes et une seconde des régates, etc. Voir Inventaire général, Antoine Le Bas (réd.), op. cit., p. 34-37.

51. Arch. dép. Essonne, 3526. Inventaire général, Antoine Le Bas (réd.), Philippe Ayrault (phot.), Juvisy-sur-orge. Un territoire et des réseaux, Lyon, Lieux-Dits, à paraître fin 2007, Cahiers du patrimoine.

52. Thomas Deschamps, op. cit., p. 83.

53. L'association, fondée en 1876 par des exilés des régions annexées en 1870 par la Prusse, s'installe sur un terrain de trois hectares en bord de Marne en 1889. Voir Inventaire général, Isabelle Duhau (réd.), Stéphane Asseline (phot.), Nogent et Le Perreux, l'eldorado en bord de Marne, Paris, APPIF, 2005, Images du patrimoine $n^{\circ} 237$, p. 90.

54. Association créée en 1908. Voir Thomas Deschamps. op. cit., p. 93.

55. La baignade, créée en 1898, était auparavant privée. Voir Thomas Deschamps, op. cit., p. 48-49.

56. La municipalité communiste inaugure la plage municipale populaire le $1^{\mathrm{er}}$ août. Le bulletin municipal du $1^{\mathrm{er}}$ octobre suivant relate l'événement : «Les municipalités communistes [font] les plus grands efforts pour l'hygiène et la santé de l'enfance et de la population. Les travailleurs ont 
obtenu de haute lutte les congés payés, la semaine de 40 heures, nous devons leur procurer ce qu'il faut pour passer agréablement les loisirs, reposer leur corps et vivifier les poumons empoisonnés par les poussières des usines. C'est ce qu'a pensé la Municipalité de Neuilly-surMarne. » Voir Thomas Deschamps, op. cit., p. 98-99 et 150.

57. À partir de la seconde moitié du XIX ${ }^{\mathrm{e}}$ siècle, des investisseurs privés urbanisèrent la banlieue en multipliant les lotissements sans se préoccuper des équipements et aménagements sanitaires nécessaires et placèrent bien des municipalités dans une situation d'urgence.

58. Thomas Deschamps, op. cit., p. 50-51.

59. En bord de Marne notamment, la baignade «sauvage " demeure longtemps une pratique fréquente, malgré son interdiction, si l'on en croit les fameux clichés de Willy Ronis ou les souvenirs de François Cavanna, racontés avec truculence dans Les Ritals (1979) : « En semaine, le jeudi ou pendant les vacances, la Marne est à nous, les nez sales. On plonge dès qu'on voit une péniche qui se pointe au tournant, on nage à fond de train pour agripper le petit canot de sauvetage qui est accroché derrière [...] ou des fois on se faufile à l'USM, le club sportif où les mecs barbotent dans leurs trente-trois mètres sous surveillance, pas le droit de s'éloigner, sifflet. On se glisse parmi ces cons branques, c'est le tremplin qui nous intéresse, on se paie des périlleux avant, des sauts de l'ange, pas spécialement impec mais on se marre. » Voir Inventaire général, Isabelle Duhau (réd.), Stéphane Asseline (phot.), Nogent et Le Perreux, op. cit., p. 72-73.

60. Thomas Deschamps, op. cit., p. 80-81.

61. Les deux vice-présidents sont E. Élefant de la plage de l'Isle-Adam et A. Richard, des bains Deligny. Les autres établissements adhérents sont : Au Banc de sable, L'île rouge à Saint-Maurice; le Bain Royal, quai des Tuileries à Paris ; la Plage bleue à Valenton; la plage de Champigny-sur-Marne ; les bains Convert, 15, boulevard de la Marne à Nogentsur-Marne ; la plage de Gournay-sur-Marne ; la baignade municipale de Joinville-le-Pont ; la plage de Meaux-Trilport; la plage de Melun; la plage de la Varenne-Saint-Hilaire, à Saint-Maur-des-Fossés ; la plage de Villennes-Medan et la plage du Lys-Chantilly à Boransur-Oise.

62. Jusqu'au XVIII ${ }^{\text {e }}$ siècle terme médical pour qualifier un délire provoqué par une affection cérébrale.

63. Terme de médecine vieilli désignant un médicament qui rend le sang moins épais.

64. Première édition de l'Encyclopédie, ou Dictionnaire Raisonné des Sciences, des Arts et des Métiers, op. cit.

65. La Natation naturelle à l'homme et l'art de nager, suivi de considérations sur les traitements hydrothérapiques et sur l'effet hygiénique des bains froids et des bains chauds, $3^{\mathrm{e}}$ édition, Paris, 1876.

66. Eugène Briffault. op. cit. p. 98.

67. Cette appellation perdure bien après que les tarifs aient augmenté. Voir Eugène Briffault, op. cit., p. 34 .

68. Les Bains de Paris..., op. cit. p. 81.

69. «J'avais pour habitude d'aller tous les matins au bain flottant de Rima, entre le petit jardin du Palais royal et l'extrémité de la douane. J'avais pris pour une heure, entre sept et huit heures, une "sirène ", c'est-à-dire une des deux vasques réservées aux dames et suffisamment grandes pour y nager un peu [...]. Comme personne d'autre ne pouvait entrer, je ne prenais pas le soin de passer mon costume de bain. La vasque, entourée de parois de bois, couverte d'une toile gris cendré à larges bandes rouges, avait un fond de planches placées à telle profondeur que les dames de petite taille n'avaient que la tête hors de l'eau. Moi, j'avais toutes les épaules découvertes.

Oh, la belle eau émeraude mais limpide où je voyais ondoyer mes formes jusqu'aux pieds que j'avais gracieux! Parfois, un poisson tout petit et argenté me frôlait. Je nageais autant 
que la longueur de la « sirène » me le permettait; je battais l'eau des mains jusqu'à ce que l'écume blanche recouvrît le vert diaphane; je m'allongeais sur le dos, laissant baigner mes longs cheveux et tentant de rester ainsi un moment immobile; [...] je riais comme une enfant. Des ouvertures nombreuses et larges, au-dessous du niveau de l'eau laissaient entrer et passer librement l'eau. Les parois, mal jointes, si on y appliquait l'oeil, permettaient à travers les fissures de voir quelque chose du dehors : le campanile rouge de San Giorgio, une ligne de lagune où fuyaient les barques légères, un petit morceau du bain militaire qui flottait à peu de distance de ma «sirène ».

Je savais que tous les matins, à sept heures, le lieutenant Remigio allait y nager. Dans l'eau, c'était un héros : il sautait du plus haut, tête la première, repêchait une bouteille au fond, sortait de l'enceinte en passant par-dessous les cabines. J'aurais donné je ne sais quoi pour réussir à la voir, tant j'étais attirée par son agilité et par sa force.

Un matin, pendant que je regardais sur ma cuisse droite une petite tache pâle, sans doute une légère contusion qui gâtait un peu la blancheur rosée de ma peau, j'entendis à l'extérieur un bruit, comme quelqu'un qui aurait nagé rapidement. L'eau s'agita, un frisson dû à la fraîche ondulation parcourut tous mes membres et, par l'un des larges trous, entre le sol et les parois, un homme entra soudainement dans la "sirène ". Je ne criais point, je n'eus point peur. Il me sembla de marbre tant il était blanc et beau, mais une respiration profonde agitait son large thorax, ses yeux bleus brillaient, et de ses cheveux blonds tombaient des gouttes comme une pluie de perles brillantes. Debout, à demi voilé par l'eau encore frissonnante, il leva ses bras musclés et blancs : on aurait dit qu'il remerciait les dieux et disait : « Enfin! »

C'est ainsi que commença notre liaison. » Camillo Boito, Senso, carnet secret de la comtesse Livia, 1883. Boito (1836-1914), par ailleurs architecte, fut un théoricien de la restauration du patrimoine.

70. Thomas Deschamps, op. cit., p. 100.

71. Ordonnance du préfet de police de mai 1888.

72. Raymond Radiguet, Les Joues en feu, 1925.

73. Isabelle Duhau, «La doctrine naturiste du docteur Durville à Physiopolis, la cité de nature de Villennes-sur-Seine », Paris et Ile-de-France, mémoires, t. 56, 2005, p. 287-308. Un bassin de natation commença d'être réalisé dans l'ensemble des équipements sportifs de Physiopolis. Mais la proximité de la plage de Villennes, sur la même île de la Seine, l'aisance en matière de baignade en pleine eau des sportifs de la communauté et la modestie de leurs moyens entraînèrent son abandon rapide.

74. Paul Christmann, La Natation et les bains. Suivi de quelques indications sur l'art de nager, Paris, A. Picard et Kaan, 1886.

75. Encyclopédie des sports, publiée sous le patronage de l'Académie des sports et du Comité national des sports C.O.F. Paris, impr. Villain et Bar, Libr. de France, 1924 (1927).

76. Christmann explique dans son ouvrage de 1887 que l'école normale militaire de gymnastique de Joinville exerce les hommes dans la Marne, et que presque tous ceux qui y suivent les cours durant la saison d'été retournent à leur régiment sachant nager. Il s'empresse de préciser que cela ne suffit pas car tous les hommes devraient savoir nager et qu'on ne parviendra à ce but qu'en construisant des piscines de natation. Pour le bassin maçonné voir Inventaire général, Isabelle Duhau (réd.), Stéphane Asseline (phot.), Nogent et Le Perreux, op. cit., p. 73.

77. Cité dans Deux siècles d'architecture sportive à Paris, op. cit., p. 27.

78. Edmond Texier, op. cit., p. 7.

79. Au sujet de l'Isle-Adam, voir « Sport et distraction », La vie à la campagne, $1^{\text {er }}$ août 1935, p. 324 ou «Plages d'eau douce aux portes de Paris », La Vie à la campagne, $1^{\mathrm{er}}$ juin 1938, p. 235. Voir 
également «Les plages fluviales», L'Illustration, $1^{\text {er }}$ septembre 1934 pour les plages d'Élisabethville, Boran-sur-Oise, l'Isle-Adam et Beaumont-sur-Oise.

80. Sport, Eau et Soleil, $\mathrm{n}^{\circ}$ 11, juillet 1965.

81. Entité administrative ayant précédée celle de la région.

82. Les bases des boucles de la Seine à Moisson et de Val-de-Seine à Verneuil-sur-Seine dans les Yvelines, celle de Cergy-Pontoise dans le Val-d'Oise, celles de Vaires-Sur-Marne, Torcy et Jablines le long de la Marne en Seine-et-Marne et celle de Bois-le-Roi le long de la Seine dans le même département, enfin celle du Port-aux-Cerises à Draveil dans l'Essonne, également près de la Seine. Voir Jérôme Maunoury, Patrice Pourtanel, Christian Mueller, « Les bases de plein air et de loisirs d'île-de-France. Pôles structurants de la politique de loisirs », Espaces, n² 249, juin 2007, p. 17-23; Guide des bases de loisirs ; temps libre, temps forts, Paris, région Île-de-France, [2007].

83. Ministère de la jeunesse et des sports. Équipement. Piscines couvertes et en plein air, Paris, Institut pédagogique national, service d'édition et de vente des publications de l'éducation nationale, 1966.

84. Inventaire général, Isabelle Duhau (réd.), Stéphane Asseline (phot.), Nogent et Le Perreux, op. cit., p. 92.

85. Thomas Deschamps, op. cit., p. 68 et Inventaire général, Antoine Le Bas (réd), Architectures du sport, op. cit., p. 96.

86. Thomas Deschamps, op. cit., p. 64-65 et Inventaire général, Antoine Le Bas (réd), Architectures du sport, op. cit., p. 96.

87. http://www.chalons-en-champagne.net.

88. "École flottante de natation (dite bains d'Orléans), construite sur la Garonne devant Bordeaux d'après les plans et sous la direction de l'architecte J. Lafargue », Revue de l'architecture et des travaux publics, 1844, col. 490-493, pl. 25-26; La Question des bains de natation, Bordeaux, imp. de J. Belmas, [1860].

89. Alain Maureau, Bains froids et écoles de natation dans le Rhône à Avignon, du XVIII au XX $X^{\mathrm{e}}$ siècle, Avignon, chez l'auteur, 1970.

90. Voir par exemple la base de loisirs de Varennes-sur-Seine (77), relevant de la communauté de communes des Deux Fleuves ou celle du parc de Chanteraines à Villeneuve-la-Garenne, appartenant au conseil général des Hauts-de-Seine.

91. Il est constitué de huit communes, deux communautés d'agglomération, le port autonome de Paris à Bonneuil-sur-Marne et la chambre de métiers du Val de Marne.

92. «Seine-et-Marne : réouverture de la plage de Meaux », Le Moniteur, 29 juin 2007 ; "Meaux inaugure sa plage », Batiactu, 05 juillet 2007.

93. « Nager dans Paris, nouvelles piscines » Techniques et architectures, oct-nov 2005.

\section{RÉSUMÉS}

On ne se baigne plus à Paris dans la Seine depuis plus de trois siècles. Les équipements, d'abord d'éphémères installations, se perfectionnent et sont réglementés par les pouvoirs publics au fur et à mesure qu'ils se pérennisent. Les simples piquets, plantés dans le lit de la rivière et couverts d'une toile tendue, sont rapidement remplacés par des établissements formés de pontons flottants qui délimitent au centre un bassin. Puis, les vestiaires et les équipements annexes sont édifiés sur les quais des rivages avant que les bassins eux-mêmes ne soient maçonnés et 
encastrés dans la rive afin de faciliter l'épuration de l'eau.À la baignade en eau vive, née de l'attrait renaissant pour le bain d'hygiène, succède l'école de natation à la fin du XVIII ${ }^{\mathrm{e}}$ siècle. Dès les beaux jours, hommes et femmes se rendent dans leurs établissements respectifs, décence oblige, pour se rafraîchir, entretenir leur santé, pratiquer un sport, se retrouver entre amis et pour les plus jeunes, oser des jeux intrépides. Les Franciliens privés de rivages marins plébiscitent les plages fluviales, où enfin, la séparation des sexes n'est plus de mise. La région compte finalement ses établissements par dizaines. Les mieux équipés proposent hôtel, casino, restaurant ou dancing pour s'attirer toujours plus de clientèle. Cependant, dans une rivière après l'autre, à partir des années 1950, la pollution entraîne bientôt l'interdiction de se baigner et sonne le glas de ces piscines d'un genre particulier qui ont presque toutes disparu

No one has ever swum in the Seine in Paris since more than three centuries. The facilities, first short-lived structures, got improved and controlled by the authorities as they became permanent constructions. Simple stakes driven in the bed of the river and supporting a stretched canvas would be replaced by facilities composed of floating landing stages around a pool. Then changing-rooms and annexes would be erected on the banks, before the pools got built into the embankments so as to make water purification easier.The fashion of swimming in river water, aroused by the renewed interest for health bath, was followed by the one of the swimming school in the late XVIII ${ }^{\text {th }}$ century. In summertime, men and women had to have the sense of decency and so would go to separate places so as to freshen up, to keep in good fit, to do sport, to meet friends and, as for the youngest, to dare play bold games. The riverside beaches where, at last, sexual separation was no longer imposed, would prove a success with the inhabitants of the Ile-deFrance, missing the seaside shores. In the region, these facilities were in dozens in the end. The best equipped ones would include a hostel, a casino, a restaurant or a dance hall to draw an ever bigger clientele. However, since the 1950's, in one river after another, swimming has been prohibited because of the pollution, putting an end to these swimming pools of a particular kind which, for most of them, no longer exist.

Seit wenigstens drei Jahrhunderten wird in der Seine in Paris nicht mehr gebadet. An den Ufern existierten zuerst vorläufige Badeeinrichtungen, die sich allmählich in dauerhafte Anstalten verwandelten, die immer mehr gesetzliche Anforderungen berücksichtigen mussten. Bald wurden einfache, in das Flussbett gerammte Holzpfähle mit Tuchbedeckung durch Schwimmpontons ersetzt, die mittlere Becken abgrenzten. Nach dem Bau von Umkleideräumen und Nebenausstattungen an den Uferstegen wurden die Becken selber ausgemauert und in das Ufer eingebaut, um eine bessere Wasserreinigung zu ermöglichen. Nach dem Erfolg des Badens im freien Gewässer, entsprungen aus hygienischen Gründen, entwickelte sich am Ende des 18. Jahrhunderts die Begeisterung für den Schwimmunterricht. Mit den ersten Frühlingstagen begaben sich Männer und Frauen aufgrund der guten Sitten in geschlechtsgetrennte Anstalten, um sich zu erfrischen, gesund zu bleiben, einen Sport zu treiben oder Freunde zu treffen. Die Jüngeren erfreuten sich auch an waghalsigen Spielen. Da die Franciliens (Einwohner der île de France) keine Meeresstrände zur Verfügung hatten, begeisterten sie sich für ihre Flussstrände, wo schließlich keine Trennung nach Geschlecht mehr gefordert wurde. In der Pariser Gegend zählte man Dutzende solcher Bäder. Die besten Ausstattungen mit Hotel, Casino, Restaurant und Tanzsaal waren für die Kunden besonders attraktiv. In den Jahren ab 1950 führte jedoch die zunehmende Wasserverschmutzung eines Flusses nach dem anderen zum Badeverbot, so dass nun fast alle diese besonderen Badeanstalten verschwunden sind. 
INDEX

Index chronologique : XIXe siècle, XXe siècle, époque contemporaine

Mots-clés : piscine

Keywords : swimming pool

Schlüsselwörter : Schwimmbad

\section{AUTEUR}

\section{ISABELLE DUHAU}

Isabelle Duhau, née en 1963, est diplômée de l'École Boulle et de l'École du Louvre ( $1^{\mathrm{er}}$ cycle et muséologie). Elle a également soutenu une maîtrise et un DEA à l'université de Paris IV, sous la direction de Bruno Foucart. Elle est actuellement chercheur au service de l'inventaire général du patrimoine culturel de la région Île-de-France où elle travaille plus particulièrement sur le patrimoine des départements des Yvelines et du Val-de-Marne. Dans ce cadre, elle a publié ou participé à la publication de plusieurs études ainsi que de cinq volumes dans la collection nationale du ministère de la culture des Images du Patrimoine. Adresse électronique :

isabelle.duhau@îledefrance.fr 